\title{
Neuronal activity in ontogeny and oncology
}

Humsa Venkatesh ${ }^{1,2}$ and Michelle Monje 1,3,4,5 $^{\text {. }}$

1 Department of Neurology, Stanford University School of Medicine, Stanford, CA

2 Cancer Biology Graduate Program, Stanford University School of Medicine, Stanford, CA

3 Department of Pediatrics, Stanford University School of Medicine, Stanford, CA

4 Department of Pathology, Stanford University School of Medicine, Stanford, CA

5 Institute for Stem Cell Biology and Regenerative Medicine, Stanford University, Stanford, CA

Corresponding author:

Michelle Monje MD PhD

265 Campus Drive, G3077

Stanford, CA 94305

mmonje@stanford.edu

\begin{abstract}
The nervous system plays a central role in regulating the stem cell niche in many organs and thereby critically modulates development, homeostasis and plasticity. A similarly powerful role for neural regulation of the cancer microenvironment is emerging. Neurons promote the growth of cancers of the brain, skin, prostate, pancreas and stomach. Parallel mechanisms shared in development and cancer suggest that neural modulation of the tumor microenvironment may prove a universal theme, although the mechanistic details of such modulation remain to be discovered for many malignancies. Here, we review what is known about the influences of active neurons on stem cell and cancer microenvironments across a broad range of tissues and discuss emerging principles of neural regulation of development and cancer.
\end{abstract}

\section{The neural regulation of development and cancer}

Direct growth-promoting effects of active neurons in the tumor microenvironment have now been described for an increasing list of cancers, and tumor innervation is emerging as an important therapeutic target. For a broad range of cancers, the neural influences on development of the 
corresponding normal tissue and the expression of receptors for neural molecules such as neurotransmitters on the tumor cells suggest that neural signaling promotes growth. However, as lessons from development suggest, neuronal influences could, conversely, promote cancer differentiation and involution. Here we will consider the functions of neuronal activity in tissue development, homeostasis and plasticity, together with the emerging roles for active neurons in tumor initiation and growth.

Since the influence of neuronal activity in development is best studied in the nervous system itself, we will begin there, as many of the mechanisms hold true in the development of non-nervous system organs as well. Recurrent themes of neural influences in development and cancer include the actions of neurotransmitters and neurotrophins in healthy and malignant tissue growth and differentiation; these mechanisms emerge as a common thread throughout the organ systems discussed below. Detailed studies of neuronal activity-dependent growth, facilitated by modern experimental tools, are also uncovering novel and unexpected mechanisms. Our understanding of neural influences on both nervous and non-nervous system organs is in its infancy. We will review what is known (and what remains to be discovered) about the influences of neurons in development and in the cancer microenvironment, organizing the breadth of the currently available information from a developmental perspective by tissue type (germ layer) and organ system.

\section{The role of neurons in ectodermal tissues}

\section{Neurodevelopment and cancers of the CNS}

The role of neuronal activity in the refinement of nervous system structure and function has long been appreciated [1-3]. Electrical activity shapes the earliest stages of brain organogenesis, as well as the behavior of persistent populations of neural precursor cells in the childhood and adult brain (Text Box 1). While the cells of origin for brain cancers remain an area of intense research and debate, it is generally accepted that the most malignant forms of brain tumors, including pediatric and adult high-grade gliomas, primitive neuroectodermal tumors (PNETs), medulloblastomas and ependymomas arise from and phenotypically resemble neural precursor cell (NPC) populations (see Glossary). Medulloblastoma subtypes are thought to arise from distinct cerebellar neuronal precursor populations or ventricular zone stem cells [4-9], high-grade gliomas from glial precursors (gliogenic stem cells, pre-oligodendroglial progenitor cells (preOPCs) or OPCs)) [10-14], and ependymomas from radial glial cells [15]. The microenvironmental 
factors regulating the proliferation, survival and differentiation of NPC populations thus bears potential relevance to the behavior of their malignant counterparts. Given the putative origins of these tumors, we will focus on the physiological role of neuronal activity on the proliferation, differentiation and survival of neural stem and precursor cells and the recapitulation of these mechanisms in central nervous system malignancies.

Electrical activity profoundly influences central nervous system (CNS) development, including neural induction [16], neural stem and precursor cell proliferation, migration, differentiation, neuronal survival, synaptogenesis, oligodendrogenesis and myelination. Canonical neuronal activity- synapse-mediated depolarization of neurons- triggers calcium influx and subsequent calcium-dependent signaling that can affect a range of cellular functions [17-19]. However, even before neurons have matured or synapses have formed, patterned waves of electrical activity are crucial to neurodevelopment. Prior to maturation and generation of classical action potentials, depolarizing neurotransmitters are released non-synaptically and calcium transients are found in all parts of the nervous system [20,21]. During embryonic development, gap junctions composed of connexin-26 (Cx26) and connexin-43 (Cx43) couple ventricular zone stem cells (radial glial cells) and NPCs into adjacent, radially arrayed clusters, particularly during the period of intense cortical neurogenesis $[22,23]$. These coupled clusters form functional units and microenvironmental cues sensed by one or more cells can thus affect the entire cluster. Depolarization and the resultant calcium transients occur synchronously throughout the cluster. Gap junctions similarly couple postnatal NPCs [24] as well as mature astrocytes [25]. Elegant recent studies showed that, like NPC populations, glioblastoma (GBM) cells form an interconnected network that similarly depends upon $\mathrm{Cx} 43$ gap junction protein-mediated communication through which calcium waves can propagate [26]. Activity-regulated growth signaling might be communicated through such interconnected cancer cells as it is in normal NPCs or astrocytes.

In mature neurons, propagation of depolarization down the axon via voltage-gated sodium channels results in synapse-mediated communication between cells. Neurotransmitters released during synaptic transmission are rapidly cleared by perisynaptic astrocytes in the healthy mature brain [27]. Synaptic release of neuropeptides also mediates communication between some classes of neurons. Growth factors such as the neurotrophin BDNF (see Text Box 2) can also be secreted in an activity-dependent fashion. The effects of neuronal activity on development and neural plasticity are also mediated indirectly by activity-dependent changes in other cells of the 
microenvironment, such as activity-dependent synaptic pruning by microglia [28]. Below, we will discuss influences of neuronal activity on neuronal and glial development as well as the corresponding malignancies.

\section{Neurogenesis and neuroepithelial tumors}

While exposure of neurogenic precursors to depolarizing neurotransmitters generally promotes generation of neurons, the effects of membrane depolarization and exposure to specific neurotransmitters on the proliferation and differentiation of NPCs varies with cell populations and with neurotransmitter types.

Early in embryonic development, paracrine non-synaptic neurotransmitter release causes ventricular zone NSC depolarization via ionotropic glutamate (AMPA/kainate) and GABA receptors [29]. Glutamate and GABA are depolarizing/excitatory neurotransmitters in immature neural stem cells; while GABA is the chief inhibitory neurotransmitter in the mature CNS, GABA results in depolarization in immature NPCs due to a high intracellular chloride concentration [30]. Exposure of NSCs to GABA or glutamate during embryonic neurogenesis results in decreased proliferation [29]. In contrast, glutamate stimulates the proliferation of restricted neuronal progenitors (neuroblasts) during prenatal forebrain neurogenesis, either via AMPA/kainate receptors (in cortical neuroblasts) or via NMDA receptors (in striatal neuroblasts) $[31,32]$.

Postnatal SVZ neurogenesis is regulated by various neurotransmitters including glutamate, GABA, dopamine, serotonin and acetylcholine. Glutamate promotes SVZ-derived neuroblast survival via NMDA receptor signaling [33]. GABA, released from differentiating neuroblasts, results in depolarization and decreased NSC proliferation [34]. Both serotonin and dopamine promote NPC proliferation in the SVZ [35-37]. A recent study identified a novel population of subventricular zone cholinergic neurons that extensively innervate the postnatal SVZ niche [38]. Optogenetic experiments (control of neuronal activity using light and neuronal expression of light-responsive microbial opsins, for review see [39]) demonstrate that activity of these cholinergic subependymal neurons promotes NPC proliferation and neuroblast generation via local acetylcholine release [38]. In the postnatal hippocampus, neuronal activity promotes the differentiation of proliferating NSCs into mature granule cell neurons via a calcium channel-dependent induction of a proneural gene expression program [40]. Postnatal hippocampal NSCs sense circuit activity largely via depolarizing GABAergic inputs [41,42]. In addition, GABAergic inputs from parvalbumin- 
positive interneurons also promote neuroblast survival [42]. In the hippocampus, glutamatergic and serotonergic signaling similarly promote neurogenesis [40,43-45].

Like the neurogenic precursor cells of the cerebrum, cerebellar GCPs are also depolarized by glutamate and by GABA via kainate and $\mathrm{GABA}_{\mathrm{A}}$ receptors, respectively [46]. The effect of depolarization on GCPs is not yet entirely clear [47]. In contrast, glutamatergic signaling through the metabotropic glutamate receptor mGluR4 decreases GCP proliferation and promotes differentiation [48]. Conversely, glutamatergic signaling through mGluR5 stimulates the proliferation of retinal neuronal precursors, emphasizing the differential effects of neurotransmitter-mediated membrane depolarization in various precursor populations [49].

The effects of elevated neuronal activity on tumors that arise from and are similar to neurogenic NPCs, such as medulloblastoma and PNETs, are difficult to predict given the diverse responses of NPC populations to depolarization and neurotransmitter exposure. The role of neuronal activity in the microenvironment of these tumors has also not been explicitly studied to date. Medulloblastoma is a disease that parses into four molecular subtypes, termed SHH, Wnt, Group 3 (Myc-amplified) and Group 4 [50,51]. A majority of human medulloblastoma samples examined express metabotropic glutamate receptors and mGluR4 expression correlates inversely with histological anaplasia, dissemination and clinical outcome [52]. mGluR4 agonism, analogous to its role in normal GCPs, reduces proliferation of human medulloblastoma cell lines in vitro and decreases tumor progression in vivo in genetic mouse models of SHH subtype [52]. Group 3 tumors exhibit up-regulated expression of the $\alpha 5$ subunit of the GABA receptor [53], but the functional roles of GABAergic signaling in Group 3 and other subtypes of medulloblastoma are not yet clear. Direct modulation of glutamatergic and GABAergic neuronal activity in the medulloblastoma microenvironment is needed to ascertain the role of neurons and to identify possible novel therapeutic strategies.

\section{Gliogenesis and gliomas}

Neuronal activity promotes the proliferation and subsequent differentiation of myelin-forming precursor cells. Decreased neuronal activity in the optic nerve, achieved via optic nerve transection or infusion of tetrodotoxin (blocker of voltage-gated sodium channels), results in decreased OPC proliferation [54]. Optogenetically elevated neuronal activity in the premotor circuit of awake, behaving animals results in brisk proliferation of OPCs and earlier precursors (Figure 2), followed 
by differentiation of the recently divided OPCs into oligodendrocytes and myelin remodeling [55]. In contrast, astroglial cells do not proliferate in response to elevated neuronal activity [55]. Activity-dependent OPC proliferation and subsequent differentiation occurs in both juvenile and adult rodent brains [55], and these activity-dependent changes in myelin-forming cells, termed "myelin plasticity" or "adaptive myelination", positively influences neurological function $[55,56]$, indicating a newly-appreciated dimension of neuroplasticity along which neuronal activity modulates brains structure and function throughout life.

The molecular mechanisms of adaptive myelination that mediate changes in OPCs remain an area of active research. A number of possible mechanisms were suggested by elegant in vitro studies, including activity-regulated adenosine and astrocyte-derived LIF [57-59]. Neuregulin-ErbB3 signaling and Bdnf signaling may also play roles in activity-regulated responses of myelin forming cells [60,61]. Additionally, OPCs express a range of neurotransmitter receptors and form bona fide synapses with neurons in both grey and white matter [62-65]. GABAergic interneurons synapse onto OPCs and, like other precursor cell populations, GABA is depolarizing in OPCs due to high intracellular $\mathrm{Cl}$ - concentrations [66]. The precise role of glutamatergic or GABAergic axoglial synapses in OPC proliferation, differentiation or migration during innate and during activitydependent myelination remains to be elucidated. Recently, it was shown that GABA decreases perinatal OPC proliferation and promotes oligodendrocyte differentiation, indicating a key functional role in development [67].

The central role that neuronal activity plays in promoting the growth, survival and functional refinement of normal brain development suggests that parallel roles in the biology of brain cancer would be advantageous to tumor growth and progression. This may be particularly true for gliomas given their similarity to gliogenic NPCs [55]. To test the role of neuronal activity in the glioma microenvironment, we recently used in vivo optogenetic stimulation of the premotor cortex in the context of a patient-derived orthotopic xenograft derived from a pediatric cortical GBM. Elevated premotor cortical projection neuronal activity resulted in a circuit-specific increase in glioma cell proliferation and overall increase in tumor growth in vivo (Figure 2) [68]. To test the role of activity-regulated secreted factors in activity-dependent glioma growth, we collected the conditioned media from acute cortical slices of varying activity levels (optogentically stimulated slices with $20 \mathrm{~Hz}$ neuronal firing rate; slices exhibiting spontaneous neuronal activity; and slices electrically silenced by tetrodotoxin) and exposed a range of patient-derived cultures derived from 
clinically and molecularly distinct high-grade glioma types to these conditioned media. Secreted factors regulated by neuronal activity increased the proliferation and growth of 9 out of 10 patientderived high-grade gliomas, including pediatric HGGs (diffuse intrinsic pontine glioma (DIPG), pediatric cortical GBM) and adult HGGs (GBM and anaplastic oligodendroglioma). Further biochemical, proteomic and functional analyses revealed that activity-regulated secretion of Bdnf and, unexpectedly, a synaptic adhesion protein called neuroligin-3 (Nlgn3) mediates this growthpromoting effects [68]. While Bdnf is known to promote OPC proliferation and myelination [69], the role of Nlgn3 in normal myelination is not yet clear, but these findings in glioma may help to elucidate molecular mechanisms of healthy myelin development and plasticity. Secreted Nlgn3 binds to an unidentified glioma cell binding partner and subsequently activates PI3K-mTOR signaling and proliferation [68]. Additionally, exposure to Nlgn3 results in a positive feed-forward loop that upregulates Nlgn3 expression, which may promote autocrine/paracrine effects. Accordingly, NLGN3 expression in human GBM correlates inversely with overall survival [68]. Subsequent work is needed to understand the potential roles that other neuronal subtypes may have on glioma growth. Further, it should be noted that the slice experimental platform described did not allow for evaluation of local effects of neurotransmitters or other molecules that are rapidly cleared from the microenvironment and cannot be detected in conditioned media but may play a key role in vivo.

Responsiveness of glioma cells to neurotransmitters has been appreciated for some time and has been considered to date largely in the context of non-synaptic paracrine/autocrine secretion of glutamate from cancer cells. Adult GBM cells secrete copious amounts of glutamate in vitro [70] and glutamate levels are increased in the tumor tissue and brain parenchyma surrounding GBM tumors in vivo [71]. Glutamate release from tumor cells can be excitotoxic to neurons in the tumor vicinity $[70,72]$ and gliomas that secrete higher levels of glutatmate exhibit a growth advantage in rodent models [72]. Glutamate effects on cell proliferation, survival and migration/invasion can be mediated by calcium-permeable AMPA receptor stimulation [73,74]. Further, gliomas increase the excitability of the neural circuit in which they are growing (Figure 1), an effect that is mediated in part by glutamate secretion [75,76]. Additional mechanisms by which gliomas promote increased neuronal activity and stimulate further growth, remain to be elucidated.

The direct effects of GABA on glioma cells are less clear. Examining a panel of patient-derived glioma cultures, one study found that functional $\mathrm{GABA}_{\mathrm{A}}$ receptors were variably expressed and 
tended to be present in lower grade gliomas (grade II and III astrocytomas) and in oligodendrogliomas rather than GBM (grade IV) [77]. In some patient-derived tumor cells, GABA was depolarizing while in others it was hyperpolarizing; intratumoral heterogeneity also dictates that some cells within a given tumor are depolarized and others are hyperpolarized [77]. Other studies examining a patient-derived GBM culture found evidence of functional $\mathrm{GABA}_{\mathrm{A}}$ receptors in a subset of cells $(\sim 12 \%)$, for which GABA and GABA agonists, such as diazepam and etomidate, were depolarizing [78]. The functional effects of GABAergic signaling in glioma are yet to be clarified, but given the effects of GABA on glial precursor cells development [67], it is conceivable that GABA could promote glioma differentiation.

Adult GBMs also express serotonin (5-HT(7)) [79] and dopamine receptors [80,81]. A recent screen of patient-derived GBM cultures against a library of neurochemical compounds identified agents modulating serotonergic, dopaminergic and cholinergic signaling, with agents blocking the dopamine receptor DRD4 particularly effective at reducing GBM cell proliferation and survival via disruption of autophagy [81]. Further supporting the concept that dopamine receptors may be a useful therapeutic target, an earlier study found that knock-down or pharmacological inhibition of D2DR dopamine receptor reduced tumor growth in patient-derived GBM cell cultures [80]. While more work is needed to elucidate the role of serotonergic signaling in glioma, a reassuring retrospective analysis of selective serotonin re-uptake inhibitor (SSRI) use in patients with both GBM and depression found no indication that SSRI and subsequent increase in microenvironmental serotonin levels affects survival [82]. Targeting neurotransmitters receptors in glioma may prove a useful therapeutic strategy.

\section{Neuronal growth factors in neurodevelopment and cancer progression}

The neurotrophins (Text Box 2) are secreted proteins that promote survival, growth, development and plasticity in the central and peripheral nervous systems. The survival of embryonic cortical neurons depends on expression of BDNF [83]. Activity-regulated $\mathrm{Ca}++$ influx through voltagegated channels promotes the binding of calcium-responsive transcription factors CREB and CaRF to regulatory elements in $B D N F$ and increased BDNF expression [84,85]. Neuronal activity also regulates BDNF secretion [86,87]. Neuronal expression and secretion of BDNF influences diverse processes, from increased NPC proliferation and survival [88], to neural circuit development [89] or to long term potentiation (LTP) [90]. In the developing cerebellum, BDNF promotes survival of 
immature GCPs in the external granule layer, while NT-3 acts on more mature cells in the internal granule layer [91]. In the oligodendroglial lineage, BDNF regulates OPC proliferation and myelination [69] as well as regeneration after demyelinating injury [92-94]. It should be noted that multiple cells types express and secrete neurotrophins, so neurotrophin signaling does not necessarily indicate involvemnet of a neuron.

The relevance of the NGF and GDNF families of neurotrophins to cancer and the potential growth advantage of maintaining functional neurons in the cancer microenvironment was suggested by the initial isolation of NGF from mouse sarcomas and GDNF from a rat glioma [95,96]. In glioma, BDNF promotes proliferation and survival through TrkB (NTRK2)-mediated signaling [68,97,98]. Consistent with this, genomic aberrations affecting the TrK genes (NTRK1, 2 and 3) are well described in human gliomas. Activating fusions of NTRK1, 2 or 3 are found in $\sim 40 \%$ of infant HGG [99]; NTRK2 fusions in $\sim 3 \%$ of pediatric pilocytic astroctyomas [100]; infrequent NTRK1 fusions in $\sim 0.5 \%$ of adult GBMs [101]; and amplification of NTRK1 or NTRK2 in $\sim 50 \%$ of DIPGs [102]. The therapeutic utility of targeting BDNF signaling in gliomas remains to be tested, but the frequency of activating mutations in pediatric HGGs and its proliferative effect indicate that it may be an important target.

The roles of the neurotrophins in medulloblastomas and other neuroectodermal brain tumors are complex and incompletely understood. Medulloblastomas express both NT-3 and TrkC; high TrkC expression levels correlate favorably with prognosis [103]. This is consistent with the role of NT-3 in differentiating granule cells [91] and reflects either a biological activity of TrkC signaling in medulloblastoma or differential expression of $\mathrm{TrkC}$ attributable to a different cell of origin or molecular subtype. Higher expression levels of TrkA in neuroblastoma, a primitive neuroectodermal tumor of the peripheral autonomic nervous system, also corresponds to favorable prognosis [104,105]. In the developing human cerebellum, p75NTR is expressed in the EGL and co-localizes with the proliferation marker Ki67; a subset of medulloblastoma tissue samples also exhibit p75NTR expression [106]. Recent studies showed that p75NTR is expressed predominantly in the SHH subgroup [107], but the functional consequence of this remains to be determined. BDNF and TrkB signaling effects in medulloblastoma are even less clear, with one study reporting exposure to BDNF and reduced viability in human medulloblastoma cell lines [108]; but another study reporting a similar effect with TrkB inhibition [109]. The function of neurotrophin signaling in ependymomas is also an area that requires investigation. In a cohort of 
pediatric ependymomas and low-grade astrocytomas, CSF levels of BDNF were increased [110]. The p75NTR receptor is more highly expressed in supratentorial ependymoma compared to infratentorial ependymoma [111]. A comprehensive evaluation of neurotrophin receptor expression across a range of human brain tumors using modern gene expression databases, followed by functional testing of neurotrophin signaling in faithful experimental models, would shed considerable light on this promising set of possible therapeutic targets.

Beyond neurotrophins, other growth factors are also secreted by neurons. For example, ventral forebrain neurons secrete sonic hedgehog (Shh) ligand, which signals to postnatal NPCs in the ventral SVZ and regulates neurogenesis of ventrally-derived olfactory bulb neuronal subtypes [112]. Similarly, Purkinje neurons in the developing cerebellum are the source of SHH ligand which drives the proliferation of GCPs in the EGL [113], instructs cerebellar astrocyte subtype specification [114], and stimulates the proliferation of SHH subtype medullobalstoma. Thus, neurons secrete a variety of neurotransmitters, neuropeptides and protein signaling ligands that affect the behaviour or normal and malignat cells.

\section{Outside the CNS: the role of peripheral nerves in development and cancer}

Skin

The peripheral nervous system (Text Box 3) elaborates extensions into every tissue in the body. Innervation occurs very early in cutaneous morphogenesis and correlates closely with the development of epidermal structures [115,116]. For example, sensory innervation precedes hair follicle development [116]; with sensory nerves innervating the follicle secreting Shh ligand [117] and follicular stem cells responding to Hh signaling. Similarly, the development and maintenance of Merkel cells, receptor cells of epithelial origin in the skin, are directly supported by sensory innervation $[118,119]$. Peripheral nerves also instruct cutaneous arterial patterning via nervederived VEGF-A and CXCL12 [120,121]. Tissue homeostasis in the epidermis is largely regulated by maintenance and proliferation of local stem cells and shares signaling mechanisms with epidermal morphogenesis [122]. Accordingly, wound healing is influenced by nerve-derived Shh ligand secretion, which supports the stem cell response [117].

Innervation appears to promote the growth of basal cell carcinoma (BCC), a cancer driven by dysregulation of Hh signaling. Peterson et al. reported that stem cells of the follicular and touch dome epithelium are the cells of origin of BCC, and that Shh-ligand expressing sensory nerves 
innervating the touch dome epithelium drive BCC progression. Ablation of these Hh-secreting nerves suppresses BCC tumor growth [123]. Thus, in skin, neuronal regulation of Hh signaling links neuron-mediated stem cell mechanisms of homeostasis to those of tumor cell proliferation.

The contributions of innervation to other forms of skin cancers are not well studied. Melanoma, the most aggressive form of skin cancer with a particular propensity to metastasize to brain, expresses a range of both NGF family neurotrophin receptors and ligands, and autocrine/paracrine neurotrophin signaling promotes the proliferation and migration of melanoma cells [124-126]. The role of epithelial innervation in primary melanoma and in CNS metastatic lesions remains to be explored experimentally.

\section{The role of neurons in mesodermal tissues}

\section{Bone Marrow and Blood}

The bone marrow (BM) niche is a classic example of local microenvironmental regulation of stem cell survival, proliferation, and differentiation. This niche includes blood vessels, stromal cells and sympathetic nerve fibers that work cooperatively to regulate hematopoiesis. Careful regulatory control is exerted on hematopoetic stem cell (HSC) quiescence and activation in response to stress or damage [127]. Innervation regulates quiescence and maintenance of the stem cell pool via TGF- $\beta$ secreted by non-myelinating Schwann cells (peripheral glial cells) that ensheath sympathetic nerves in the BM. Sympathetic denervation results in loss of TGF- $\beta$ signaling and depletion of the BM HSC population [128]. Migration of newly generated blood cells out of the $\mathrm{BM}$ niche is another key step in blood cell production and can be stimulated by granulocyte-colony stimulating factor (G-CSF) [129]. This migratory response to G-CSF is linked to the chemotactic interaction of CXCR4/CXCL12 within the niche [130]. An important study by Katayama et al. demonstrated that genetically-engineered mice with poor nerve conduction have a dramatic reduction in the egress of the HSCs in response to G-CSF and other known migration-stimulating cytokines.. The migratory response of HSCs appears to be directly regulated by the sympathetic nervous system, since it is impaired by pharmacological or genetic ablation of adrenergic signaling and can be rescued with $\beta_{2}$ adrenergic agonists [131].

The nervous system further regulates blood homeostasis via circadian oscillations of adrenergic activity and oscillatory release of HSCs. Norepinephrine and dopamine levels vary with circadian rhythms in the BM niche, with peak levels observed at night, a pattern disrupted by 
sympathectomy [132]. Further linking circadian secretion of norepinephrine to the the cyclic release of HSCs, sympathetic nervous system (SNS) signaling via $\beta_{3}$-receptors on BM stromal cells down-regulates CXCL12 and results in HSC release [133]. The role of SNS in the retention of HSCs suggests a role in blood homeostasis and regeneration by promoting HSC circulation during periods of rest. Supporting a role of the nervous system in regeneration, chemotherapyinduced bone marrow nerve injury hinders hematopoietic regeneration, while nerve regeneration promotes recovery [134].

Perivascular mesenchymal stem and progenitor cells (MSPCs) are also a key component of the $\mathrm{BM}$ niche and maintain the HSC population through expression of growth and retention factors including CXCL12, SCF, and ANGPT1 [135,136]. These MPSCs associate with sympathetic nerve fibers near blood vessels to form what is termed the neuroreticular complex [137,138]. Innervation regulates proliferation and differentiation of MSPCs, as proliferation of quiescent MSPCs increases after sympathectomy, and osteoblastic differentiation decreases in response to $\beta 3$-adrenergic receptor signaling [135]. Together, these findings emphasize the importance of the nervous system in stem cell behavior within the BM niche.

While role of the nervous system in hematological cancer progression remains to be elucidated, neurotrophin signaling does have an established role. A recently described neurotrophin neurotrophin-1 (NNT-1)/B cell- stimulating factor-3 (BSF-3) - was linked to growth and survival of multiple myeloma (MM) in vitro [139-141]. Neurotrophins have also been implicated in MM progression. MM cells secrete BDNF and express high levels of its receptor TrkB; and BDNF triggers activation of survival pathways including Akt and MAPK [142]. In the BM niche, BDNF is expressed by osteoblasts, megakaryocytes, and endothelial cells [143], suggesting that non-neuronal cells may also contribute to MM through neurotrophin signaling. This reciprocal interaction between $\mathrm{MM}$ and the BM microenvironment may also lead to worsened bone disease by stimulating osteoclasts and enhancing bone resorption through the BDNF/TRKB axis [144].

\section{Bone}

Bone homeostasis is regulated by a balance of formation by osteoblasts and resorption by osteoclasts. The bone is extensively innervated and tissue turnover is heavily influenced by neural signals. The sympathetic nervous system influences both osteoblast and osteoclast activity. Chemically sympathectomized mice demonstrate a decrease in osteoblastic activity, with reduced 
uptake of collagen precursors and deposition of the bone matrix [145,146]. Both $\beta$-adrenergic and glutamatergic signaling influences osteoblast function and bone remodeling. Leptin (antiosteogenic peptide hormone) plays a crucial role in bone homeostasis, and leptin-deficient mice have an increased rate of bone formation [147]. Takeda et al. demonstrated that $\beta$-adrenergic receptors modulate osteoblast differentiation downstream of leptin signaling. Correspondingly, $\beta$ adrenergic agonists decrease bone mass, while $\beta$-adrenergic antagonists increase bone mass [148], underscoring a role for the nervous system in bone homeostasis. Other neurotransmitters also influence bone precursor cell function and bone growth. Osteoblasts secrete glutamate in an autocrine/paracrine manner [149] and express both ionotropic and metabotropic glutamate receptors. Glutamatergic signaling through ionotrophic glutatmate receptors (AMPA and NMDA receptors) stimulates osteoblast ERK signaling, osteoblast maturation, and promotes bone formation [150]. NMDA receptor stimulation in osteoblasts induces c-fos and jun expression, both of which promote osteoblast proliferation and have been linked to osteosarcoma initiation [151].

The role that innervation plays in mediating bone growth and homeostasis suggests that it could play a similarly prominent role in bone cancer pathogenesis. Present evidence indicates that a variety of receptors for neural molecules are expressed by a range of bone cancer cells. Ewing sarcoma and osteosarcoma cells express $\beta$-adrenergic receptors [152]. Both normal osteoblasts and osteosarcoma cells also express several neuropeptide receptors including VIP receptor, substance P receptor, Y receptor, and calcitonin gene-related peptide receptor [153]. A variety of glutamate receptors are also expressed in osteosarcoma cells [154]. The gene encoding mGluR4 was identified in a genome-wide association (GWAS) study of osteosarcoma [155]. mGluR4 was found to be expressed in a significant percentage of both osteosarcoma $(\sim 20 \%)$ and benign giant cell tumors of bone $(\sim 40 \%)$, and expression correlated positively with overall survival in osteosarcoma [156]. The function of NMDA-mediated or AMPA-mediated glutamatergic signaling in bone cancers remians to be determined. Adrenergic, dopaminergic and VIP-mediated signaling all promote glycogenolytic metabolism in Ewing sarcoma cells, suggesting a role in cancer metabolism [157,158]. Adrenergic signaling induces expression of IL-6 and IL-11 in osteoblasts and osteosarcoma cells that stimulates osteoclast production [159] and may thereby promote bone remodeling and facilitate tumor mass expansion.

\section{Muscle}


Motor neuron innervation regulates skeletal muscle development and homeostasis. In drosophila, innervation is critical for muscle patterning and myoblast proliferation [160]. During embryonic development, innervation is also necessary for myoblast differentiation into mature myofibers; and comparisons of denervated and toxin-inactivated muscle indicate that innervation provides both electrical signals and trophic support [161]. Spinal cord explants promote the growth and maintenance of skeletal myofibers in vitro [162], indicating that trophic support is also important for homeostasis. Muscle atrophy that occurs following denervation is thus due not only to disuse, but also to loss of these trophic signals. is one motor neuron-derived secreted signal that promotes neuro-muscular junction (NMJ) synaptogenesis [163,164]. NT-3 is also expressed by motor neurons during development and its TrkC receptor is expressed by skeletal muscle fibers [165]

Little is known about the role of innervation in rhabdomyosarcomas. In studies using models of murine sarcoma, in which tumor cells are implanted into thigh muscles, dopamine injections inhibited endothelial progenitor cell mobilization and slowed tumor growth. [166,167]. The possible roles of motor neuron innervation in rhabdomyosarcoma remain to be explored.

\section{The role of neurons in endodermal tissues}

\section{Submandibular salivary gland}

Like the systems discussed above, glandular development also depends on the activity of neurons (Figure 2). The role of innervation in glandular organogenesis is best studied in salivary glands. Knox et al. showed that during organogenesis,epithelial cell progenitor populations are maintained by direct parasympathetic innervation. Using explant cultures of submandibular gland, they demonstrated that removal of the parasympathetic ganglion (PSG) reduced growth of the normal salivary gland epithelium, as evidenced by a decrease in the number of end buds (acini). Antagonism of acetylcholine production or the associated muscarinic receptor also had an inhibitory effect. Markers of epithelial progenitor cells were decreased after removal of the PSG, suggesting that cholinergic innervation is necessary to the maintenance of the glandular epithelium by maintaining the progenitor cell population [168]. In a different study, the same group identified the neuronal-derived vasoactive intestinal peptide (VIP)/cAMP/PKA pathway and CFTR as key players during salivary gland tubulogenesis [169]. Thus, parasympathetic innervation coordinates multiple aspects of organogenesis throughout gland development. Illustrating the reciprocal developmental roles of peripheral nerves and their target organs, nerve outgrowth from mouse 
submandibular ganglion is stimulated by the local epithelium ([170], Figure 2A), and it has been suggested that salivary epithelial cells may recruit the growth promoting effects of the PSG by secreting neurturin to promote nerve outgrowth [169].

Salivary gland growth, maintenance, and function are also regulated by autonomic nerves [171]. Both parasympathetic cholinergic signaling through muscarinic receptors, and sympathetic signaling through adrenergic receptors modulates saliva secretion. Parasympathetic nerve-derived VIP may also play a role [172]. Though all mechanisms involved have not yet been elucidated, it is known that after denervation the gland undergoes degeneration and atrophy, suggesting that innervation is also crucial for maintenance [171,173-175]. While the role of innervation in salivary gland tumors is unexplored, innervation has been clearly demonstrated to play a key role in cancers of other glandular organs such as the prostate and pancreas, as discussed below.

\section{Prostate}

Prostate cancer is among the malignancies for which a growth-promoting role of innervation is best established. The microanatomical relationship of cancer cells with neural elements in the microenvironment first suggested an important role for nerves. A variety of cancers, including prostate cancer, exhibit perineural invasion (PNI), first described by Bastsakis as the invasion of tumor cells in, around, and through the nerves [176]; for review, see [177]. Perineural space invasion and diameter are linked to prostate adenocarcinoma aggressiveness [178-180]. Analysis of gene expression in reactive stroma from grade 3 prostate cancer samples showed high expression of genes involved in the neurogenesis, axonogenesis, and synaptogenesis, including GDNF, NOTCH1, FGF19, GLI2, and CDK5 [181]. More recently, evidence of prostate-cancer related axonogenesis has come to light and correlates clinically with recurrence and aggressiveness [182]. Axonogenesis, the process of sending out axons to reach new targets based on microenvironmental axon guidance molecules (semaphorins, netrins, ephrins, cell adhesion molecules, neurotransmitters, and other growth factors), is normal in neural development and plasticity (for a review see [183]). Cancer-related axonogenesis is a more recently described phenomenon, best illustrated by work from Ayala's group. An instructive role of prostate cancer in this exuberant axonal ingrowth into the tumor microenvironment was demonstrated in co-culture models of prostate cancer cell lines and dorsal root ganglia neurons. In this model, cancer cells promoted DRG neurite outgrowth and then proceeded to migrate along these nascent neurites, 
mimicking perineural invasion. Compared to controls of tumor cells alone or DRG neurons alone, when cultured together more tumor cell colonies formed and a higher degree of neurite outgrowth was observed, suggesting that DRG neurons and prostate cancer cells promote reciprocal growth ([184], Figure 2). Prostate cancer-induced axonogenesis has since been demonstrated in vivo [185], and, as discussed below, has also been described in other malignancies such as pancreatic cancer. Nerve density correlates with prostate cancer cell proliferation and with the expression of proteins involved in survival and hormonal regulation pathways in human radical prostatectomy specimens [186]. Another group has shown that proNGF (precursor to nerve growth factor and known to induce axonogenesis) was overexpressed in prostate cancer specimens in comparison to $\mathrm{BPH}$ samples. Levels of proNGF correlated positively with the Gleason score (a measure of prostate cancer aggressiveness). Additionally, co-culture of PC12 neuron-like cells or 50B11 neurons with PC-3 cells showed increased neurite outgrowth in vitro, which could be blocked with an antibody against proNGF [187]. The observation that cancers promote axonal sprouting in the tumor microenvironment supports the overarching concept that increased innervation is advantageous to cancer progression (Figure 3B).

The prostate gland is densely innervated and regulated by the autonomic nervous system. Parasympathetic input (via the pelvic nerve) and sympathetic input (via the hypogastric nerve) regulate growth of the prostate [188,189]. [190]. Adrenergic innervation is a key regulator, as rodent sympathectomy of the hypogastric nerve causes a reduction in overall weight of the prostate [189]. Adrenergic agonism with phenylephrine results in benign prostatic hyperplasia (BPH), providing additional evidence that sympathetic signaling contributes to prostate growth [191,192]. Cholinergic nerve fibers are present in both stromal and glandular epithelial compartments of the prostate [193-195]. While the role of cholinergic signaling in normal prostate growth remains to be elucidated [195,196], muscarinic acetylcholine receptors are highly expressed in both the normal prostate as well as in some prostate cancer cells, suggesting that cancer cells may take advantage of the same growth signals.

Indeed, autonomic neurotransmitters are the best-understood mechanism of innervation-induced prostate cancer progression. Cholinergic signaling through muscarinic receptors causes prostate cancer cell proliferation in vitro [197,198]. Adrenergic signaling promotes prostate tumor migration in vitro and in vivo [199]. In prostate xenograft models, lumbar lymph node metastases increased with exposure to norepinephrine, which was blocked with propranolol (B-blocker). Both 
treatments had no effect on primary tumor growth [200]. Elegant recent work by the Frenette group elucidated multiple facets of autonomic innervation in prostate tumors. Using xenograft and genetic models, they demonstrated that sympathetic and parasympathetic innervation contribute to distinct aspects of cancer progression [185]. Adrenergic signaling via sympathetic innervation is important during initial stages of cancer development, and surgical or chemical sympathectomy or genetic deletion of stromal $\beta 2$ - and $\beta 3$-adrenergic receptors results in poor tumor engraftment and growth. In contrast, cholinergic signaling via parasympathetic innervation promotes cancer spread. Disruption of parasympathetic signaling by pharmacological inhibition or genetic deletion of stromal type 1 muscarinic receptor reduced tumor invasion and metastasis. Similarly, in stratified human radical prostatectomy specimens; adrenergic fibers are present in surrounding normal prostate tissues, while cholinergic fibers infiltrate the tumor. Higher density of nerve fibers is associated with poor clinical outcome [185]. Taken together, these data indicate a crucial role for innervation in prostate cancer growth and progression and identify both adrenergic and muscarinic signaling as promising therapeutic targets.

\section{Pancreas}

Neurons in the microenvironment of pancreatic adenocarcinoma have been widely studied in the context of perineural invasion and histopathologically-apparent alterations in local nerves. Like prostate cancer, pancreatic adenocarcinoma exhibits increased overall nerve density [201], suggesting cancer-induced axonogenesis. While $90 \%$ of patients show intrapancreatic perineural invasion, those with additional extrapancreatic nerve plexus involvement have decreased survival [202]. Intrapancreatic, extratumoral perineural invasion also correlates negatively with postoperative survival [202,203]. Nerve plexus invasion is now regarded as one of the most important prognostic factors in invasive ductal carcinoma of the pancreas [204].

Invasion promoted by peripheral nerves was demonstrated in systems of isolated myenteric plexus neurons (MP) or dorsal root ganglia (DRG) neurons co-cultured with pancreatic cancer cells. Both neuron types increased cell invasion through a 3D extracellular matrix gel [205]. The molecular mediators of perineural invasion are coming to light, although it remains to be determined whether and which factors are derived directly from the nerves. Neurotrophins, including NGF and artemin (a GFL neurotrophic factor), are increased in pancreatic adenocarcinoma compared to the normal pancreas [206]. Artemin acts through GFRalpha3/RET receptors to influence cancer spread along 
pancreatic nerves [207]. Clinical studies examining the expression of GDNF and its receptor tyrosine kinase RET, linked both to pancreatic cancer proliferation and metastasis, invasion, and survival [5,208]. Secretion of GDNF and downstream activation of RET-Ras-MAPK pathway induces migration along nerve fibers [209]. The NGF/TrkA pathway has also been implicated in perineural invasion by prompting nerve hyperplasia [210]. Although both receptors of NGF, TrkA and p75NTR, are associated with perineural invasion, only TrkA is associated with poor prognosis [211]. Consistent with its known function in pro-apoptotic signaling in some molecular contexts, p75NTR is associated with a favorable prognosis [211]. NGF is thus thought to exert dual effects in pancreatic cancer, dependent on patterns of receptor expression.

Importantly, cancer-associated nerve alterations begin in the pre-malignant stages of pancreatic cancer. Stopczynski et al. monitored changes in innervation, neurotrophin expression and perineural invasion in genetically-engineered mouse models of pancreatic ductal adenocarcinoma and observed increased nerve density quite early in the disease course, when only pancreatic intraepithelial neoplasia was present. Increases in neurotrophic factor ligand and receptor expression were also observed from the earliest stages of malignancy. These dramatic changes in intrapancreatic nerve density and neurotrophin mRNA expression correlate with the early onset of pain, evidenced by pain-related decreases in exploratory behavior and nociceptive gene expression in sensory ganglia [212]. Work correlating neuroplastic changes in pancreatic nerves with the onset of pancreatic cancer suggest a critical role of innervation in cancer initiation and progression and helps explaining the early and prominent pain experienced by pancreatic cancer patients.

\section{Gastrointestinal system}

The extensive innervation of the gastrointestinal tract by the enteric nervous system (ENS), the vagus nerve and sympathetic nerves, controls organ function. After initial colonization, the neural crest-derived ENS precursor cells migrate, proliferate, and differentiate to form two layers of ganglia comprising the submucosal and myenteric plexi [213,214]. These processes are regulated through a variety of signaling pathways, including but not limited to GDNF/RET, endothelin3/EDNRB, as well as classical axon path-finding molecules like netrins and semaphorins [215223]. Development of the ENS, as well as autonomic innervation in other organs, is particularly dependent on GFL neurotrophin signaling, as loss of Gdnf, neurturin (Nrtn), RET or GFR $\alpha$ RET 
co-receptor (Gfral or Gfra2) genes results in insufficient development and severe GI dysfunction [222,224-228].

In the gastric epithelium, homeostasis is regulated by a balance of Wnt and Notch signaling, which promote proliferation of gastric epithelial stem cells, and bone morphogenetic protein BMP4 signaling, which influences differentiation [229]. Innervation promotes the growth of gastric stem cells via cholinergic stimulation of Wnt signaling, as illustrated by co-culture of gastric stem cells with enteric neurons. Pharmacological inhibition of muscarinic signaling suppressed Wnt signaling and gastric organoid growth in a manner dependent on muscarinic receptor subtype 3 (Chrm3) expression [230]. Recent elegant work further demonstrated that acetylcholine induces LGR5+ enteric stem cell proliferation via YAP-dependent modulation of Wnt signaling [231]. These findings, together with additional data supporting a trophic effect of muscarinic signaling on gastric stem cell proliferation [232] help to explain the long-standing observation that vagotomy results in severe gastric atrophy after denervation [233]. Similarly, cholinergic nerves regulate the maintenance and proliferation of progenitor cells in the intestinal epithelium [234]. Non-synaptic paracrine secretion of serotonin from ENS neurons promotes growth and turnover of intestinal mucosal epithelium through the activation of 5-HT(2A) receptors on cholinergic submucosal neurons, which then provide muscarinic innervation to intestinal epithelium [235]. Likewise in the liver, proliferation of hepatic progenitor cells is regulated by the vagus nerve, most likely via binding of acetylcholine to the Chrm3 receptor [236].

Like the effects on normal enteric stem cells, autonomic innervation also plays a role in enteric tumor growth. Chemical denervation with benzalkonium chloride (BAC) was found to block development of gastric tumors in a rodent model [237], a finding in contrast to early studies indicating that vagotomy promotes the development of gastric cancers [238,239]. Zhao et al. subsequently demonstrated that denervation at a preneoplastic stage inhibits tumorigenesis in a genetic mouse model of gastric cancer, and that denervation after onset slowed tumor growth [230]. Gene profiling analyses in the denervated gastric tumors identified Wnt and Notch signaling as regulators of tumor growth [230], both of which are known to be key players in normal gastric stem cell development and regeneration [240]. Similarly in the intestine, knock out of Chrm3 in a genetic model of small intestinal neoplasia reduced tumor number, size and $\beta$-catenin expression [241], consistent with the effects of muscarinic signaling on normal intestinal stem cell 
proliferation. Enteric tumors encourage axonogenesis and increase innervation of the tumor microenvironment by secreting NGF [231]. Acetylcholine released by the recruited nerves drives cancer growth through increased Wnt signaling and further stimulates NGF release in a feedforward cycle [231]. Strikingly, upregulated NGF secretion and subsequently increased enteric innervation is sufficient to initiate tumorigenesis [231]. Like neuronal regulation of Hedgehog signaling discussed above in brain and skin, neuronal regulation of Wnt signaling via muscarinic innervation in both the stomach and intestine represents an unexpected mechanism of neural regulation of signaling pathways widely implicated in both development and cancer. These shared neural signaling mechanisms suggest the cancer is commandeering normal trophic mechanisms of tissue homeostasis and regeneration. Enteric innervation emerges as a therapeutic target and anticholinergic agents as potentially useful drugs.

\section{Concluding Remarks}

Key insights pointing towards the importance of neural elements in the cancer microenvironment have come from histopathological observations. The importance of neuron-glioma interactions has long been suggested by a classic histopathological feature of high-grade gliomas called "perineuronal satellitosis", characterized by microanatomical clustering of glioma cells around neuronal somata [242]. Similarly, the clinical significance of perineural invasion and increased axonal density within the tumor microenvironment of a number of malignancies has highlighted the potential roles of innervation in cancer progression. Advanced microscopy techniques are now shedding new light on further microanatomical relationships central to cancer biology, for example the interconnected network formed by Cx43-dependent glioma "microtubes" revealed by multiphoton laser-scanning microscopy [26]. Application of exciting new imaging techniques such as EM tomography, CLARITY [243,244] and super-resolution microscopy may reveal further structural aspects of neuron-cancer cell interactions in various tissue types.

Nervous system activity strongly modulates the function of stem and precursor cells, influencing organ development, maintenance, plasticity and regeneration in a diverse range of tissues. These neuron-mediated developmental mechanisms are recapitulated or hijacked in malignancy. While the roles of neural elements in cancer growth are becoming increasingly clear for a number of malignancies, considerable work remains to be done in others. In all cases, the mechanisms 
mediating neuronal influences on cancer growth and progression are likely incompletely understood (see Outstanding Questions). Presently unexplored are the roles neurons may play not only in cancer growth, but also initiation. Use of modern neuroscience techniques for modulating neuronal activity in both the central and peripheral nervous systems, such as in vivo optogenetics or chemogenetics, in the context of cancer models that faithfully represent the human disease will be enormously informative. Already, our emerging appreciation of neural contributions to cancer growth and progression has elucidated novel therapeutic avenues for cancers of the brain, prostate, stomach, pancreas and skin. As our mechanistic understanding grows, neural targets in the cancer microenvironment may prove crucial for effective disease control.

Acknowledgements: We thank Chamelli Jhappan, Paul Frenette and Sam Pfaff for organizing the Nerves in Cancer meeting held at the National Cancer Institute in March 2015 and to all participants for galvanizing and thought-provoking discussions, and to Sarah Knox for critical reading of this manuscript. We gratefully acknowledge support from the National Institutes of Neurological Disease and Stroke (R01NS092597 to M.M.), the National Cancer Institute (1F31CA200273 to H.V.), the V Foundation (to M.M.), the California Institute for Regenerative Medicine (RN3-06510 to M.M.) and the Department of Defense (NF140075 to M.M.). We thank Sarah Chen for the figure illustrations.

\section{References:}

1 Wiesel, T.N. and Hubel, D.H. (1965) Comparison of the effects of unilateral and bilateral eye closure on cortical unit responses in kittens. J. Neurophysiol. 28, 1029-40

2 WIESEL, T.N. and HUBEL, D.H. (1963) EFFECTS OF VISUAL DEPRIVATION ON MORPHOLOGY AND PHYSIOLOGY OF CELLS IN THE CATS LATERAL GENICULATE BODY. J. Neurophysiol. 26, 978-93

3 WIESEL, T.N. and HUBEL, D.H. (1963) SINGLE-CELL RESPONSES IN STRIATE CORTEX OF KITTENS DEPRIVED OF VISION IN ONE EYE. J. Neurophysiol. 26, 1003-17

4 Schüller, U. et al. (2008) Acquisition of granule neuron precursor identity is a critical determinant of progenitor cell competence to form Shh-induced medulloblastoma. Cancer Cell 14, 123-34 
5 Yang, Z.-J. et al. (2008) Medulloblastoma can be initiated by deletion of Patched in lineage-restricted progenitors or stem cells. Cancer Cell 14, 135-45

6 Gibson, P. et al. (2010) Subtypes of medulloblastoma have distinct developmental origins. Nature 468, 1095-9

7 Kawauchi, D. et al. (2012) A mouse model of the most aggressive subgroup of human medulloblastoma. Cancer Cell 21, 168-80

8 Pei, Y. et al. (2012) An animal model of MYC-driven medulloblastoma. Cancer Cell 21, $155-67$

9 Lin, C.Y. et al. (2016) Active medulloblastoma enhancers reveal subgroup-specific cellular origins. Nature 530, 57-62

10 Monje, M. et al. (2011) Hedgehog-responsive candidate cell of origin for diffuse intrinsic pontine glioma. Proc. Natl. Acad. Sci. U. S. A. 108, 4453-4458

11 Liu, C. et al. (2011) Mosaic analysis with double markers reveals tumor cell of origin in glioma. Cell 146, 209-221

12 Persson, A.I. et al. (2010) Non-Stem Cell Origin for Oligodendroglioma. Cancer Cell 18, $669-682$

13 Sugiarto, S. et al. (2011) Asymmetry-defective oligodendrocyte progenitors are glioma precursors. Cancer Cell 20, 328-340

14 Galvao, R.P. et al. (2014) Transformation of quiescent adult oligodendrocyte precursor cells into malignant glioma through a multistep reactivation process. Proc. Natl. Acad. Sci. U. S. A. 111, E4214-23

15 Taylor, M.D. et al. (2005) Radial glia cells are candidate stem cells of ependymoma. Cancer Cell 8, 323-35

16 Webb, S.E. et al. (2005) Calcium transients and neural induction in vertebrates. Cell Calcium 37, 375-85

17 Deisseroth, K. et al. (1998) Translocation of calmodulin to the nucleus supports CREB phosphorylation in hippocampal neurons. Nature 392, 198-202

18 Bito, H. et al. (1996) CREB phosphorylation and dephosphorylation: a $\mathrm{Ca}(2+)$ - and stimulus duration-dependent switch for hippocampal gene expression. Cell 87, 1203-14

19 Deisseroth, K. et al. (1996) Signaling from synapse to nucleus: postsynaptic CREB phosphorylation during multiple forms of hippocampal synaptic plasticity. Neuron 16, 89- 
101

20 Wong, R.O. et al. (1995) Early functional neural networks in the developing retina. Nature 374, 716-8

21 Corlew, R. et al. (2004) Spontaneous, synchronous electrical activity in neonatal mouse cortical neurones. J. Physiol. 560, 377-90

22 Bittman, K.S. and LoTurco, J.J. (1999) Differential regulation of connexin 26 and 43 in murine neocortical precursors. Cereb. Cortex 9, 188-95

23 LoTurco, J.J. et al. (1991) Initial expression and endogenous activation of NMDA channels in early neocortical development. J. Neurosci. 11, 792-9

24 Marins, M. et al. (2009) Gap junctions are involved in cell migration in the early postnatal subventricular zone. Dev. Neurobiol. 69, 715-30

25 Yamamoto, T. et al. (1990) LM and EM immunolocalization of the gap junctional protein connexin 43 in rat brain. Brain Res. 508, 313-9

26 Osswald, M. et al. (2015) Brain tumour cells interconnect to a functional and resistant network. Nature 528, 93-8

27 Rothstein, J.D. et al. (1996) Knockout of glutamate transporters reveals a major role for astroglial transport in excitotoxicity and clearance of glutamate. Neuron 16, 675-86

28 Schafer, D.P. et al. (2012) Microglia sculpt postnatal neural circuits in an activity and complement-dependent manner. Neuron 74, 691-705

29 LoTurco, J.J. et al. (1995) GABA and glutamate depolarize cortical progenitor cells and inhibit DNA synthesis. Neuron 15, 1287-98

30 Rivera, C. et al. (1999) The K+/Cl- co-transporter KCC2 renders GABA hyperpolarizing during neuronal maturation. Nature 397, 251-5

31 Luk, K.C. et al. (2003) Glutamate promotes proliferation of striatal neuronal progenitors by an NMDA receptor-mediated mechanism. J. Neurosci. 23, 2239-50

32 Luk, K.C. and Sadikot, A.F. Glutamate and regulation of proliferation in the developing mammalian telencephalon. Dev. Neurosci. 26, 218-28

33 Platel, J.-C. et al. (2010) NMDA receptors activated by subventricular zone astrocytic glutamate are critical for neuroblast survival prior to entering a synaptic network. Neuron $65,859-72$

34 Liu, X. et al. (2005) Nonsynaptic GABA signaling in postnatal subventricular zone 
controls proliferation of GFAP-expressing progenitors. Nat. Neurosci. 8, 1179-87

35 Van Kampen, J.M. et al. (2004) Induction of neurogenesis in the adult rat subventricular zone and neostriatum following dopamine D3 receptor stimulation. Eur. J. Neurosci. 19, 2377-87

36 Banasr, M. et al. (2004) Serotonin-induced increases in adult cell proliferation and neurogenesis are mediated through different and common 5-HT receptor subtypes in the dentate gyrus and the subventricular zone. Neuropsychopharmacology 29, 450-60

37 O'Keeffe, G.C. et al. (2009) Dopamine-induced proliferation of adult neural precursor cells in the mammalian subventricular zone is mediated through EGF. Proc. Natl. Acad. Sci. U. S. A. 106, 8754-9

38 Paez-Gonzalez, P. et al. (2014) Identification of distinct ChAT+ neurons and activitydependent control of postnatal SVZ neurogenesis. Nat. Neurosci. 17, 934-942

39 Deisseroth, K. (2015) Optogenetics: 10 years of microbial opsins in neuroscience. Nat. Neurosci. 18, 1213-1225

40 Deisseroth, K. et al. (2004) Excitation-neurogenesis coupling in adult neural stem/progenitor cells. Neuron 42, 535-52

41 Tozuka, Y. et al. (2005) GABAergic excitation promotes neuronal differentiation in adult hippocampal progenitor cells. Neuron 47, 803-15

42 Song, J. et al. (2013) Parvalbumin interneurons mediate neuronal circuitry-neurogenesis coupling in the adult hippocampus. Nat. Neurosci. 16, 1728-30

43 Cameron, H.A. et al. (1995) Regulation of adult neurogenesis by excitatory input and NMDA receptor activation in the dentate gyrus. J. Neurosci. 15, 4687-92

44 Santarelli, L. (2003) Requirement of Hippocampal Neurogenesis for the Behavioral Effects of Antidepressants. Science (80-. ). 301, 805-809

45 Malberg, J.E. et al. (2000) Chronic antidepressant treatment increases neurogenesis in adult rat hippocampus. $J$. Neurosci. 20, 9104-10

46 Dave, K.A. and Bordey, A. (2009) GABA increases Ca2+ in cerebellar granule cell precursors via depolarization: implications for proliferation. IUBMB Life 61, 496-503

47 Fiszman, M.L. et al. (1999) GABA induces proliferation of immature cerebellar granule cells grown in vitro. Brain Res. Dev. Brain Res. 115, 1-8

48 Canudas, A.M. et al. (2004) PHCCC, a specific enhancer of type 4 metabotropic 
glutamate receptors, reduces proliferation and promotes differentiation of cerebellar granule cell neuroprecursors. J. Neurosci. 24, 10343-52

49 Zhang, Z. et al. (2016) Activation of type 5 metabotropic glutamate receptor promotes the proliferation of rat retinal progenitor cell via activation of the PI-3-K and MAPK signaling pathways. Neuroscience 322, 138-151

50 Cho, Y.-J. et al. (2011) Integrative genomic analysis of medulloblastoma identifies a molecular subgroup that drives poor clinical outcome. J. Clin. Oncol. 29, 1424-30

51 Northcott, P.A. et al. (2010) Medulloblastoma Comprises Four Distinct Molecular Variants. J. Clin. Oncol. 29, 1408-1414

52 Iacovelli, L. et al. (2006) Pharmacological activation of mGlu4 metabotropic glutamate receptors inhibits the growth of medulloblastomas. J. Neurosci. 26, 8388-97

53 Sengupta, S. et al. (2014) a5-GABAA receptors negatively regulate MYC-amplified medulloblastoma growth. Acta Neuropathol. 127, 593-603

54 Barres, B.A. and Raff, M.C. (1993) Proliferation of oligodendrocyte precursor cells depends on electrical activity in axons. Nature 361, 258-260

55 Gibson, E.M. et al. (2014) Neuronal Activity Promotes Oligodendrogenesis and Adaptive Myelination in the Mammalian Brain. Science 344, 1-16

56 McKenzie, I.A. et al. (2014) Motor skill learning requires active central myelination. Science (80-. ). 346, 318-322

57 Demerens, C. et al. (1996) Induction of myelination in the central nervous system by electrical activity. Proc. Natl. Acad. Sci. U. S. A. 93, 9887-92

58 Stevens, B. et al. (2002) Adenosine: a neuron-glial transmitter promoting myelination in the CNS in response to action potentials. Neuron 36, 855-68

59 Ishibashi, T. et al. (2006) Astrocytes promote myelination in response to electrical impulses. Neuron 49, 823-32

60 Lundgaard, I. et al. (2013) Neuregulin and BDNF induce a switch to NMDA receptordependent myelination by oligodendrocytes. PLoS Biol. 11, e1001743

61 Makinodan, M. et al. (2012) A critical period for social experience-dependent oligodendrocyte maturation and myelination. Science 337, 1357-60

62 Bergles, D.E. et al. (2000) Glutamatergic synapses on oligodendrocyte precursor cells in the hippocampus. Nature 405, 187-91 
63 Káradóttir, R. et al. (2005) NMDA receptors are expressed in oligodendrocytes and activated in ischaemia. Nature 438, 1162-6

64 Kukley, M. et al. (2007) Vesicular glutamate release from axons in white matter. Nat. Neurosci. 10, 311-20

65 Ziskin, J.L. et al. (2007) Vesicular release of glutamate from unmyelinated axons in white matter. Nat. Neurosci. 10, 321-30

66 Lin, S. and Bergles, D.E. (2004) Synaptic signaling between GABAergic interneurons and oligodendrocyte precursor cells in the hippocampus. Nat. Neurosci. 7, 24-32

67 Zonouzi, M. et al. (2015) GABAergic regulation of cerebellar NG2 cell development is altered in perinatal white matter injury. Nat. Neurosci. 18, 674-82

68 Venkatesh, H.S. et al. (2015) Neuronal Activity Promotes Glioma Growth through Neuroligin-3 Secretion. Cell 161, 803-16

69 Wong, A.W. et al. (2013) Oligodendroglial expression of TrkB independently regulates myelination and progenitor cell proliferation. J. Neurosci. 33, 4947-57

70 Ye, Z.C. and Sontheimer, H. (1999) Glioma cells release excitotoxic concentrations of glutamate. Cancer Res. 59, 4383-91

71 Behrens, P.F. et al. (2000) Extracellular glutamate and other metabolites in and around RG2 rat glioma: an intracerebral microdialysis study. J. Neurooncol. 47, 11-22

72 Takano, T. et al. (2001) Glutamate release promotes growth of malignant gliomas. Nat. Med. 7, 1010-5

73 Ishiuchi, S. et al. (2007) Ca2+-permeable AMPA receptors regulate growth of human glioblastoma via Akt activation. J. Neurosci. 27, 7987-8001

74 Ishiuchi, S. et al. (2002) Blockage of $\mathrm{Ca}(2+)$-permeable AMPA receptors suppresses migration and induces apoptosis in human glioblastoma cells. Nat. Med. 8, 971-8

75 Buckingham, S.C. et al. (2011) Glutamate release by primary brain tumors induces epileptic activity. Nat. Med. 17, 1269-74

76 Campbell, S.L. et al. (2012) Human glioma cells induce hyperexcitability in cortical networks. Epilepsia 53, 1360-70

77 Labrakakis, C. et al. (1998) Functional GABA A receptors on human glioma cells. Eur. J. Neurosci. 10, 231-238

78 Babateen, O. et al. (2015) Etomidate, propofol and diazepam potentiate GABA-evoked 
GABAA currents in a cell line derived from human glioblastoma. Eur. J. Pharmacol. 748, $101-7$

79 Mahé, C. et al. (2004) Functional expression of the serotonin 5-HT7 receptor in human glioblastoma cell lines. Br. J. Pharmacol. 143, 404-10

80 Li, J. et al. (2014) Genome-wide shRNA screen revealed integrated mitogenic signaling between dopamine receptor D2 (DRD2) and epidermal growth factor receptor (EGFR) in glioblastoma. Oncotarget 5, 882-93

81 Dolma, S. et al. (2016) Inhibition of Dopamine Receptor D4 Impedes Autophagic Flux, Proliferation, and Survival of Glioblastoma Stem Cells. Cancer Cell 29, 859-73

82 Caudill, J.S. et al. (2011) Selective serotonin reuptake inhibitors, glioblastoma multiforme, and impact on toxicities and overall survival: the mayo clinic experience. Am. J. Clin. Oncol. 34, 385-7

83 Ghosh, A. et al. (1994) Requirement for BDNF in activity-dependent survival of cortical neurons. Science 263, 1618-23

84 Tao, X. et al. (2002) A calcium-responsive transcription factor, CaRF, that regulates neuronal activity-dependent expression of BDNF. Neuron 33, 383-95

85 Tao, X. et al. (1998) Ca2+ influx regulates BDNF transcription by a CREB family transcription factor-dependent mechanism. Neuron 20, 709-26

86 Androutsellis-Theotokis, A. et al. (1996) The depolarisation-induced release of [125I]BDNF from brain tissue. Brain Res. 743, 40-8

87 Goggi, J. et al. (2003) The control of [125I]BDNF release from striatal rat brain slices. Brain Res. 967, 201-9

88 Bartkowska, K. et al. (2007) Trk signaling regulates neural precursor cell proliferation and differentiation during cortical development. Development 134, 4369-80

89 Hong, E.J. et al. (2008) A biological function for the neuronal activity-dependent component of Bdnf transcription in the development of cortical inhibition. Neuron 60, $610-24$

90 Korte, M. et al. (1995) Hippocampal long-term potentiation is impaired in mice lacking brain-derived neurotrophic factor. Proc. Natl. Acad. Sci. U. S. A. 92, 8856-60

91 Segal, R.A. et al. (1992) Changes in neurotrophin responsiveness during the development of cerebellar granule neurons. Neuron 9, 1041-52 
92 VonDran, M.W. et al. (2011) Levels of BDNF impact oligodendrocyte lineage cells following a cuprizone lesion. J. Neurosci. 31, 14182-90

93 Fulmer, C.G. et al. (2014) Astrocyte-derived BDNF supports myelin protein synthesis after cuprizone-induced demyelination. J. Neurosci. 34, 8186-96

94 Tsiperson, V. et al. Brain-derived neurotrophic factor deficiency restricts proliferation of oligodendrocyte progenitors following cuprizone-induced demyelination. ASN Neuro 7,

95 Cohen, S. et al. (1954) A NERVE GROWTH-STIMULATING FACTOR ISOLATED FROM SARCOM AS 37 AND 180. Proc. Natl. Acad. Sci. U. S. A. 40, 1014-8

96 Lin, L.F. et al. (1993) GDNF: a glial cell line-derived neurotrophic factor for midbrain dopaminergic neurons. Science 260, 1130-2

97 Xiong, J. et al. (2013) Mature BDNF promotes the growth of glioma cells in vitro. Oncol. Rep. 30, 2719-24

98 Lawn, S. et al. (2015) Neurotrophin signaling via TrkB and TrkC receptors promotes the growth of brain tumor-initiating cells. J. Biol. Chem. 290, 3814-24

99 Wu, G. et al. (2014) The genomic landscape of diffuse intrinsic pontine glioma and pediatric non-brainstem high-grade glioma. Nat. Genet. 46, 444-50

100 Jones, D.T.W. et al. (2013) Recurrent somatic alterations of FGFR1 and NTRK2 in pilocytic astrocytoma. Nat. Genet. 45, 927-32

101 Frattini, V. et al. (2013) The integrated landscape of driver genomic alterations in glioblastoma. Nat. Genet. 45, 1141-9

102 Grasso, C.S. et al. (2015) Functionally defined therapeutic targets in diffuse intrinsic pontine glioma. Nat. Med. 21, 827

103 Segal, R.A. et al. (1994) Expression of the neurotrophin receptor TrkC is linked to a favorable outcome in medulloblastoma. Proc. Natl. Acad. Sci. U. S. A. 91, 12867-71

104 Nakagawara, A. et al. (1993) Association between high levels of expression of the TRK gene and favorable outcome in human neuroblastoma. N. Engl. J. Med. 328, 847-54

105 Suzuki, T. et al. (1993) Lack of high-affinity nerve growth factor receptors in aggressive neuroblastomas. J. Natl. Cancer Inst. 85, 377-84

106 Barnes, M. et al. (2009) Expression of p75NTR in fetal brain and medulloblastomas: evidence of a precursor cell marker and its persistence in neoplasia. J. Neurooncol. 92, $193-201$ 
107 Liang, L. et al. (2015) Characterization of novel biomarkers in selecting for subtype specific medulloblastoma phenotypes. Oncotarget 6, 38881-900

108 Schmidt, A.L. et al. (2010) BDNF and PDE4, but not the GRPR, regulate viability of human medulloblastoma cells. J. Mol. Neurosci. 40, 303-10

109 Thomaz, A. et al. (2015) BDNF/TrkB Signaling as a Potential Novel Target in Pediatric Brain Tumors: Anticancer Activity of Selective TrkB Inhibition in Medulloblastoma Cells. J. Mol. Neurosci. DOI: 10.1007/s12031-015-0689-0

110 Chiaretti, A. et al. (2004) Neurotrophic factor expression in childhood low-grade astrocytomas and ependymomas. Childs. Nerv. Syst. 20, 412-9

111 Hagel, C. et al. (2013) Supra- and infratentorial pediatric ependymomas differ significantly in NeuN, p75 and GFAP expression. J. Neurooncol. 112, 191-7

112 Ihrie, R.A. et al. (2011) Persistent sonic hedgehog signaling in adult brain determines neural stem cell positional identity. Neuron 71, 250-62

113 Wechsler-Reya, R.J. and Scott, M.P. (1999) Control of neuronal precursor proliferation in the cerebellum by Sonic Hedgehog. Neuron 22, 103-14

114 Farmer, W.T. et al. (2016) Neurons diversify astrocytes in the adult brain through sonic hedgehog signaling. Science (80-. ). 351, 849-854

115 Munger, G.T. and Munger, B.L. (1991) Differentiation of the anterior body wall and truncal epidermis and associated co-migration of cutaneous nerves and mesenchyme. Anat. Rec. 231, 261-74

116 Peters, E.M.J. et al. (2002) Developmental timing of hair follicle and dorsal skin innervation in mice. J. Comp. Neurol. 448, 28-52

117 Brownell, I. et al. (2011) Nerve-derived sonic hedgehog defines a niche for hair follicle stem cells capable of becoming epidermal stem cells. Cell Stem Cell 8, 552-65

118 English, K.B. et al. (1983) Effects of chronic denervation in type I cutaneous mechanoreceptors (Haarscheiben). Anat. Rec. 207, 79-88

119 Nurse, C.A. et al. (1984) A quantitative study of the time course of the reduction in Merkel cell number within denervated rat touch domes. Neuroscience 11, 521-33

120 Mukouyama, Y. et al. (2002) Sensory nerves determine the pattern of arterial differentiation and blood vessel branching in the skin. Cell 109, 693-705

121 Li, W. et al. (2013) Peripheral nerve-derived CXCL12 and VEGF-A regulate the 
patterning of arterial vessel branching in developing limb skin. Dev. Cell 24, 359-71

122 Blanpain, C. and Fuchs, E. (2009) Epidermal homeostasis: a balancing act of stem cells in the skin. Nat. Rev. Mol. Cell Biol. 10, 207-217

123 Peterson, S.C. et al. (2015) Basal cell carcinoma preferentially arises from stem cells within hair follicle and mechanosensory niches. Cell Stem Cell 16, 400-12

124 Sherwin, S.A. et al. (1979) Human melanoma cells have both nerve growth factor and nerve growth factor-specific receptors on their cell surfaces. Proc. Natl. Acad. Sci. U. S. A. $76,1288-92$

125 Herrmann, J.L. et al. (1993) Mediation of NGF-stimulated extracellular matrix invasion by the human melanoma low-affinity p 75 neurotrophin receptor: melanoma p 75 functions independently of trkA. Mol. Biol. Cell 4, 1205-16

126 Truzzi, F. et al. (2008) Neurotrophins and their receptors stimulate melanoma cell proliferation and migration. J. Invest. Dermatol. 128, 2031-40

127 Wilson, A. et al. (2009) Balancing dormant and self-renewing hematopoietic stem cells. Curr. Opin. Genet. Dev. 19, 461-8

128 Yamazaki, S. et al. (2011) Nonmyelinating Schwann cells maintain hematopoietic stem cell hibernation in the bone marrow niche. Cell 147, 1146-58

129 Gianni, A.M. et al. (1989) Granulocyte-macrophage colony-stimulating factor to harvest circulating haemopoietic stem cells for autotransplantation. Lancet (London, England) 2, $580-5$

130 Lévesque, J.-P. et al. (2003) Disruption of the CXCR4/CXCL12 chemotactic interaction during hematopoietic stem cell mobilization induced by GCSF or cyclophosphamide. $J$. Clin. Invest. 111, 187-196

131 Katayama, Y. et al. (2006) Signals from the sympathetic nervous system regulate hematopoietic stem cell egress from bone marrow. Cell 124, 407-21

132 Maestroni, G.J.M. et al. (1998) Neural and endogenous catecholamines in the bone marrow. Circadian association of norepinephrine with hematopoiesis? Exp. Hematol. 26, $1172-1177$

133 Méndez-Ferrer, S. et al. (2008) Haematopoietic stem cell release is regulated by circadian oscillations. Nature 452, 442-7

134 Lucas, D. et al. (2013) Chemotherapy-induced bone marrow nerve injury impairs 
hematopoietic regeneration. Nat. Med. 19, 695-703

135 Méndez-Ferrer, S. et al. (2010) Mesenchymal and haematopoietic stem cells form a unique bone marrow niche. Nature 466, 829-34

136 Frenette, P.S. et al. (2013) Mesenchymal stem cell: keystone of the hematopoietic stem cell niche and a stepping-stone for regenerative medicine. Annu. Rev. Immunol. 31, 285316

137 Kunisaki, Y. et al. (2013) Arteriolar niches maintain haematopoietic stem cell quiescence. Nature 502, 637-43

138 Yamazaki, K. and Allen, T.D. (1990) Ultrastructural morphometric study of efferent nerve terminals on murine bone marrow stromal cells, and the recognition of a novel anatomical unit: The "neuro-reticular complex." Am. J. Anat. 187, 261-276

139 Shi, Y. et al. (1999) Computational EST database analysis identifies a novel member of the neuropoietic cytokine family. Biochem. Biophys. Res. Commun. 262, 132-8

140 Burger, R. et al. (2003) Functional significance of novel neurotrophin-1/B cell-stimulating factor-3 (cardiotrophin-like cytokine) for human myeloma cell growth and survival. Br. J. Haematol. 123, 869-78

141 Senaldi, G. et al. (1999) Novel neurotrophin-1/B cell-stimulating factor-3: a cytokine of the IL-6 family. Proc. Natl. Acad. Sci. U. S. A. 96, 11458-63

142 Pearse, R.N. et al. (2005) A neurotrophin axis in myeloma: TrkB and BDNF promote tumor-cell survival. Blood 105, 4429-36

143 Labouyrie, E. et al. (1999) Expression of neurotrophins and their receptors in human bone marrow. Am. J. Pathol. 154, 405-15

144 Sun, C.-Y. et al. (2012) Brain-derived neurotrophic factor is a potential osteoclast stimulating factor in multiple myeloma. Int. J. Cancer 130, 827-36

145 Herskovits, M.S. and Singh, I.J. (1984) Effect of guanethidine-induced sympathectomy on osteoblastic activity in the rat femur evaluated by $3 \mathrm{H}$-proline autoradiography. Acta Anat. (Basel). 120, 151-5

146 Singh, I.J. et al. (1981) Autoradiographic assessment of 3H-proline uptake by osteoblasts following guanethidine-induced sympathectomy in the rat. Cell Tissue Res. 216, 215-20

147 Ducy, P. et al. (2000) Leptin Inhibits Bone Formation through a Hypothalamic Relay. Cell 100, 197-207 
148 Takeda, S. et al. (2002) Leptin Regulates Bone Formation via the Sympathetic Nervous System. Cell 111, 305-317

149 Hinoi, E. et al. (2002) Facilitation of glutamate release by ionotropic glutamate receptors in osteoblasts. Biochem. Biophys. Res. Commun. 297, 452-8

150 Lin, T.-H. et al. (2008) Regulation of the maturation of osteoblasts and osteoclastogenesis by glutamate. Eur. J. Pharmacol. 589, 37-44

151 Wang, Z.-Q. et al. (1995) c-fos-induced Osteosarcoma Formation in Transgenic Mice: Cooperativity with c-jun and the Role of Endogenous c-fos. Cancer Res. 55, 6244-6251

152 Whitsett, J.A. et al. (1983) beta-Adrenergic receptors in pediatric tumors: uncoupled beta 1-adrenergic receptor in Ewing's sarcoma. J. Natl. Cancer Inst. 71, 779-86

153 Togari, A. et al. (1997) Expression of mRNAs for neuropeptide receptors and betaadrenergic receptors in human osteoblasts and human osteogenic sarcoma cells. Neurosci. Lett. 233, 125-8

154 Kalariti, N. et al. Characterization of the glutametergic system in MG-63 osteoblast-like osteosarcoma cells. Anticancer Res. 24, 3923-9

155 Savage, S.A. et al. (2013) Genome-wide association study identifies two susceptibility loci for osteosarcoma. Nat. Genet. 45, 799-803

156 Wang, S. et al. (2016) Expression of metabotropic glutamate receptor 4 in osteosarcoma. Mol. Clin. Oncol. 4, 65-69

157 van Valen, F. and Keck, E. (1988) Induction of glycogenolysis in cultured Ewing's sarcoma cells by dopamine and beta-adrenergic agonists. J. Cancer Res. Clin. Oncol. 114, $266-72$

158 Van Valen, F. et al. (1989) Vasoactive intestinal peptide receptor regulation of cAMP accumulation and glycogen hydrolysis in the human Ewing's sarcoma cell line WE-68. Cell. Signal. 1, 435-46

159 Kondo, A. et al. (2001) Signal transduction system for interleukin-6 and interleukin-11 synthesis stimulated by epinephrine in human osteoblasts and human osteogenic sarcoma cells. Biochem. Pharmacol. 61, 319-26

160 Fernandes, J.J. and Keshishian, H. (1998) Nerve-muscle interactions during flight muscle development in Drosophila. Development 125, 1769-79

161 Sohal, G.S. and Holt, R.K. (1980) Role of innervation on the embryonic development of 
skeletal muscle. Cell Tissue Res. 210, 383-93

162 Hooisma, J. et al. (1978) Trophic support by neural explants of cultured muscle fibers. Exp. Neurol. 62, 628-46

163 Magill-Solc, C. and McMahan, U.J. (1988) Motor neurons contain agrin-like molecules. $J$. Cell Biol. 107, 1825-33

164 Gautam, M. et al. (1996) Defective neuromuscular synaptogenesis in agrin-deficient mutant mice. Cell 85, 525-35

165 Hess, D.M. et al. (2007) Localization of TrkC to Schwann cells and effects of neurotrophin-3 signaling at neuromuscular synapses. J. Comp. Neurol. 501, 465-82

166 Basu, S. et al. (2001) The neurotransmitter dopamine inhibits angiogenesis induced by vascular permeability factor/vascular endothelial growth factor. Nat. Med. 7, 569-74

167 Chakroborty, D. et al. (2008) Dopamine regulates endothelial progenitor cell mobilization from mouse bone marrow in tumor vascularization. J. Clin. Invest. 118, 1380-9

168 Knox, S.M. et al. (2010) Parasympathetic innervation maintains epithelial progenitor cells during salivary organogenesis. Science 329, 1645-7

169 Nedvetsky, P.I. et al. (2014) Parasympathetic innervation regulates tubulogenesis in the developing salivary gland. Dev. Cell 30, 449-62

170 Coughlin, M.D. (1975) Target organ stimulation of parasympathetic nerve growth in the developing mouse submandibular gland. Dev. Biol. 43, 140-58

171 Kang, J.H. et al. (2010) Parasympathectomy induces morphological changes and alters gene-expression profiles in the rat submandibular gland. Arch. Oral Biol. 55, 7-14

172 Ekström, J. et al. (1983) Vasoactive intestinal peptide evoked secretion of fluid and protein from rat salivary glands and the development of supersensitivity. Acta Physiol. Scand. 119, 169-75

173 Kyriacou, K. and Garrett, J.R. (1988) Morphological changes in the rabbit submandibular gland after parasympathetic or sympathetic denervation. Arch. Oral Biol. 33, 281-90

174 Mandour, M.A. et al. (1977) Effect of tympanic neurectomy on human parotid salivary gland. Histopathologic, Histochemical, and Clinical Study. Arch. Otolaryngol. 103, 338 41

175 Proctor, G.B. and Carpenter, G.H. (2007) Regulation of salivary gland function by autonomic nerves. Auton. Neurosci. 133, 3-18 
176 Batsakis, J.G. Nerves and neurotropic carcinomas. Ann. Otol. Rhinol. Laryngol. 94, 426-7

177 Amit, M. et al. (2016) Mechanisms of cancer dissemination along nerves. Nat. Rev. Cancer 16, 399-408

178 Maru, N. et al. (2001) Prognostic significance of the diameter of perineural invasion in radical prostatectomy specimens. Hum. Pathol. 32, 828-33

179 Villers, A. et al. (1989) The role of perineural space invasion in the local spread of prostatic adenocarcinoma. J. Urol. 142, 763-8

180 Anderson, P.R. et al. (1998) Perineural invasion and Gleason 7-10 tumors predict increased failure in prostate cancer patients with pretreatment PSA $<10 \mathrm{ng} / \mathrm{ml}$ treated with conformal external beam radiation therapy. Int. J. Radiat. Oncol. 41, 1087-1092

181 Dakhova, O. et al. (2009) Global gene expression analysis of reactive stroma in prostate cancer. Clin. Cancer Res. 15, 3979-89

182 Ayala, G.E. et al. (2008) Cancer-related axonogenesis and neurogenesis in prostate cancer. Clin. Cancer Res. 14, 7593-603

183 Dickson, B.J. (2002) Molecular mechanisms of axon guidance. Science 298, 1959-64

184 Ayala, G.E. et al. (2001) In vitro dorsal root ganglia and human prostate cell line interaction: redefining perineural invasion in prostate cancer. Prostate 49, 213-23

185 Magnon, C. et al. (2013) Autonomic nerve development contributes to prostate cancer progression. Science 341, 1236361

186 Olar, A. et al. (2014) Biologic correlates and significance of axonogenesis in prostate cancer. Hum. Pathol. 45, 1358-64

187 Pundavela, J. et al. (2014) ProNGF correlates with Gleason score and is a potential driver of nerve infiltration in prostate cancer. Am. J. Pathol. 184, 3156-62

188 Cunha, G.R. et al. (1987) The endocrinology and developmental biology of the prostate. Endocr. Rev. 8, 338-62

189 McVary, K.T. et al. (1994) Growth of the rat prostate gland is facilitated by the autonomic nervous system. Biol. Reprod. 51, 99-107

190 Swyer, G.I. (1944) Post-natal growth changes in the human prostate. J. Anat. 78, 130-45

191 Golomb, E. et al. (1998) Induction of atypical prostatic hyperplasia in rats by sympathomimetic stimulation. Prostate 34, 214-21

192 Kim, J. et al. (2009) A signaling network in phenylephrine-induced benign prostatic 
hyperplasia. Endocrinology 150, 3576-83

193 Vaalasti, A. and Hervonen, A. (1980) Autonomic innervation of the human prostate. Invest. Urol. 17, 293-7

194 Dunzendorfer, U. et al. (1976) The autonomic innervation of the human prostate. Histochemistry of acetylcholinesterase in the normal and pathologic states. Urol. Res. 4, 29-31

195 Witte, L.P.W. et al. (2008) Cholinergic innervation and muscarinic receptors in the human prostate. Eur. Urol. 54, 326-34

196 McVary, K.T. et al. (1998) Prostate innervation. Prostate. Suppl. 8, 2-13

197 Luthin, G.R. et al. (1997) Role of $\mathrm{m} 1$ receptor-G protein coupling in cell proliferation in the prostate. Life Sci. 60, 963-8

198 Rayford, W. et al. (1997) Muscarinic cholinergic receptors promote growth of human prostate cancer cells. Prostate 30, 160-6

199 Barbieri, A. et al. (2015) The stress hormone norepinephrine increases migration of prostate cancer cells in vitro and in vivo. Int. J. Oncol. 47, 527-34

200 Palm, D. et al. (2006) The norepinephrine-driven metastasis development of PC-3 human prostate cancer cells in BALB/c nude mice is inhibited by beta-blockers. Int. J. cancer $118,2744-9$

201 Ceyhan, G.O. et al. (2009) Pancreatic neuropathy and neuropathic pain--a comprehensive pathomorphological study of 546 cases. Gastroenterology 136, 177-186.e1

202 Nakao, A. et al. (1996) Clinical significance of carcinoma invasion of the extrapancreatic nerve plexus in pancreatic cancer. Pancreas 12, 357-61

203 Takahashi, T. et al. (1997) Perineural invasion by ductal adenocarcinoma of the pancreas. J. Surg. Oncol. 65, 164-70

204 Mitsunaga, S. et al. (2007) Detail histologic analysis of nerve plexus invasion in invasive ductal carcinoma of the pancreas and its prognostic impact. Am. J. Surg. Pathol. 31, 163644

205 Ceyhan, G.O. et al. (2008) Neural invasion in pancreatic cancer: a mutual tropism between neurons and cancer cells. Biochem. Biophys. Res. Commun. 374, 442-7

206 Ceyhan, G.O. et al. (2010) Nerve growth factor and artemin are paracrine mediators of pancreatic neuropathy in pancreatic adenocarcinoma. Ann. Surg. 251, 923-31 
207 Ceyhan, G.O. et al. (2006) The neurotrophic factor artemin promotes pancreatic cancer invasion. Ann. Surg. 244, 274-81

208 Zhu, Z. et al. (1999) Nerve growth factor expression correlates with perineural invasion and pain in human pancreatic cancer. J. Clin. Oncol. 17, 2419-28

209 Gil, Z. et al. (2010) Paracrine regulation of pancreatic cancer cell invasion by peripheral nerves. J. Natl. Cancer Inst. 102, 107-18

210 Ma, J. et al. (2008) Expression of nerve growth factor and tyrosine kinase receptor A and correlation with perineural invasion in pancreatic cancer. J. Gastroenterol. Hepatol. 23, $1852-9$

211 Dang, C. et al. (2006) Expression of nerve growth factor receptors is correlated with progression and prognosis of human pancreatic cancer. J. Gastroenterol. Hepatol. 21, $850-8$

212 Stopczynski, R.E. et al. (2014) Neuroplastic changes occur early in the development of pancreatic ductal adenocarcinoma. Cancer Res. 74, 1718-27

$213 \mathrm{Fu}$, M. et al. (2004) Embryonic development of the ganglion plexuses and the concentric layer structure of human gut: a topographical study. Anat. Embryol. (Berl). 208, 33-41

214 YNTEMA, C.L. and HAMMOND, W.S. (1954) The origin of intrinsic ganglia of trunk viscera from vagal neural crest in the chick embryo. J. Comp. Neurol. 101, 515-41

215 Hearn, C.J. et al. (1998) GDNF and ET-3 differentially modulate the numbers of avian enteric neural crest cells and enteric neurons in vitro. Dev. Biol. 197, 93-105

216 Heuckeroth, R.O. et al. (1998) Neurturin and GDNF promote proliferation and survival of enteric neuron and glial progenitors in vitro. Dev. Biol. 200, 116-29

217 Young, H.M. et al. (2001) GDNF is a chemoattractant for enteric neural cells. Dev. Biol. $229,503-16$

218 Natarajan, D. et al. (2002) Requirement of signalling by receptor tyrosine kinase RET for the directed migration of enteric nervous system progenitor cells during mammalian embryogenesis. Development 129, 5151-60

219 Zhu, L. et al. (2004) Spatiotemporal regulation of endothelin receptor-B by SOX10 in neural crest-derived enteric neuron precursors. Nat. Genet. 36, 732-7

220 Wang, X. et al. (2011) Analysis of the sacral neural crest cell contribution to the hindgut enteric nervous system in the mouse embryo. Gastroenterology 141, 992-1002.e1-6 
221 Jiang, Y. et al. (2003) Netrins and DCC in the guidance of migrating neural crest-derived cells in the developing bowel and pancreas. Dev. Biol. 258, 364-84

222 Gianino, S. et al. (2003) GDNF availability determines enteric neuron number by controlling precursor proliferation. Development 130, 2187-98

223 Taraviras, S. et al. (1999) Signalling by the RET receptor tyrosine kinase and its role in the development of the mammalian enteric nervous system. Development 126, 2785-97

224 Cacalano, G. et al. (1998) GFRalpha1 is an essential receptor component for GDNF in the developing nervous system and kidney. Neuron 21, 53-62

225 Sánchez, M.P. et al. (1996) Renal agenesis and the absence of enteric neurons in mice lacking GDNF. Nature 382, 70-3

226 Heuckeroth, R.O. et al. (1999) Gene targeting reveals a critical role for neurturin in the development and maintenance of enteric, sensory, and parasympathetic neurons. Neuron $22,253-63$

227 Rossi, J. et al. (1999) Retarded growth and deficits in the enteric and parasympathetic nervous system in mice lacking GFR alpha2, a functional neurturin receptor. Neuron 22, $243-52$

228 Uesaka, T. et al. (2008) Diminished Ret expression compromises neuronal survival in the colon and causes intestinal aganglionosis in mice. J. Clin. Invest. 118, 1890-1898

229 Mills, J.C. and Shivdasani, R.A. (2011) Gastric epithelial stem cells. Gastroenterology $140,412-24$

230 Zhao, C.-M. et al. (2014) Denervation suppresses gastric tumorigenesis. Sci. Transl. Med. $6,250 \mathrm{ra} 115$

231 Hayakawa, Y. et al. (2017) Nerve growth factor promotes gastric tumorigenesis through aberrant cholinergic signaling. Cancer Cell in press.

232 Aihara, T. et al. (2003) Impaired gastric secretion and lack of trophic responses to hypergastrinemia in M3 muscarinic receptor knockout mice. Gastroenterology 125, 1774 1784

233 Håkanson, R. et al. (1984) The vagus exerts trophic control of the stomach in the rat. Gastroenterology 86, 28-32

234 Lundgren, O. et al. (2011) Intestinal epithelial stem/progenitor cells are controlled by mucosal afferent nerves. PLoS One 6, e16295 
235 Gross, E.R. et al. (2012) Neuronal Serotonin Regulates Growth of the Intestinal Mucosa in Mice. Gastroenterology 143, 408-417.e2

236 Cassiman, D. et al. (2002) The vagal nerve stimulates activation of the hepatic progenitor cell compartment via muscarinic acetylcholine receptor type 3. Am. J. Pathol. 161, 52130

237 Polli-Lopes, A.C. et al. (2003) Myenteric denervation reduces the incidence of gastric tumors in rats. Cancer Lett. 190, 45-50

238 Tatsuta, M. et al. (1985) Promotion by vagotomy of gastric carcinogenesis induced by Nmethyl-N'-nitro-N-nitrosoguanidine in Wistar rats. Cancer Res. 45, 194-7

239 Tatsuta, M. et al. (1988) Effects of bilateral and unilateral vagotomy on gastric carcinogenesis induced by N-methyl-N'-nitro-N-nitrosoguanidine in Wistar rats. Int. J. Cancer 42, 414-8

240 Han, M.-E. and Oh, S.-O. (2013) Gastric stem cells and gastric cancer stem cells. Anat. Cell Biol. 46, 8-18

241 Raufman, J.-P. et al. (2011) Muscarinic receptor subtype-3 gene ablation and scopolamine butylbromide treatment attenuate small intestinal neoplasia in Apcmin/+ mice. Carcinogenesis 32, 1396-402

242 Scherer, H. (1938) Structural Development in Gliomas. Am. J. Cancer 34, 333-351

243 Chung, K. et al. (2013) Structural and molecular interrogation of intact biological systems. Nature 497, 332-7

244 Tomer, R. et al. (2014) Advanced CLARITY for rapid and high-resolution imaging of intact tissues. Nat. Protoc. 9, 1682-97

245 Hicks, S.P. and D'Amato, C.J. (1968) Cell migrations to the isocortex in the rat. Anat. Rec. 160, 619-34

246 Rakic, P. (1972) Mode of cell migration to the superficial layers of fetal monkey neocortex. J. Comp. Neurol. 145, 61-83

247 McConnell, S.K. (1985) Migration and differentiation of cerebral cortical neurons after transplantation into the brains of ferrets. Science 229, 1268-71

248 McConnell, S.K. (1988) Fates of visual cortical neurons in the ferret after isochronic and heterochronic transplantation. J. Neurosci. 8, 945-74

249 Yakovlev, Paul I., and A.-R.L. (1967) The myelogenetic cycles of regional maturation of 
the brain: Regional development of the brain in early life. Blackwell Sci. Publ.

250 Lebel, C. et al. (2012) Diffusion tensor imaging of white matter tract evolution over the lifespan. Neuroimage 60, 340-52

251 Kiessling, M.C. et al. (2014) Cerebellar granule cells are generated postnatally in humans. Brain Struct. Funct. 219, 1271-86

252 Komuro, H. et al. (2001) Mode and tempo of tangential cell migration in the cerebellar external granular layer. J. Neurosci. 21, 527-40

253 Curtis, M.A. et al. (2007) Human neuroblasts migrate to the olfactory bulb via a lateral ventricular extension. Science 315, 1243-9

254 Sanai, N. et al. (2011) Corridors of migrating neurons in the human brain and their decline during infancy. Nature 478, 382-6

255 Wang, C. et al. (2011) Identification and characterization of neuroblasts in the subventricular zone and rostral migratory stream of the adult human brain. Cell Res. 21, $1534-50$

256 Menn, B. et al. (2006) Origin of oligodendrocytes in the subventricular zone of the adult brain. J. Neurosci. 26, 7907-18

257 Xing, Y.L. et al. (2014) Adult neural precursor cells from the subventricular zone contribute significantly to oligodendrocyte regeneration and remyelination. J. Neurosci. $34,14128-46$

258 Eriksson, P.S. et al. (1998) Neurogenesis in the adult human hippocampus. Nat. Med. 4, $1313-7$

259 Spalding, K.L. et al. (2013) Dynamics of hippocampal neurogenesis in adult humans. Cell $153,1219-27$

260 Dawson, M.R.L. et al. (2003) NG2-expressing glial progenitor cells: an abundant and widespread population of cycling cells in the adult rat CNS. Mol. Cell. Neurosci. 24, 47688

261 Geha, S. et al. (2010) NG2+/Olig2+ cells are the major cycle-related cell population of the adult human normal brain. Brain Pathol. 20, 399-411

262 Young, K.M. et al. (2013) Oligodendrocyte dynamics in the healthy adult CNS: evidence for myelin remodeling. Neuron 77, 873-85

263 Peters, A. and Sethares, C. (2004) Oligodendrocytes, their progenitors and other 
neuroglial cells in the aging primate cerebral cortex. Cereb. Cortex 14, 995-1007

264 Yeung, M.S.Y. et al. (2014) Dynamics of oligodendrocyte generation and myelination in the human brain. Cell 159, 766-74

265 Peters, A. et al. (2001) Effects of age on the thickness of myelin sheaths in monkey primary visual cortex. J. Comp. Neurol. 435, 241-8

266 Park, H. and Poo, M. (2013) Neurotrophin regulation of neural circuit development and function. Nat. Rev. Neurosci. 14, 7-23

267 Meeker, R.B. and Williams, K.S. (2015) The p75 neurotrophin receptor: at the crossroad of neural repair and death. Neural Regen. Res. 10, 721-5

268 Paratcha, G. et al. (2003) The neural cell adhesion molecule NCAM is an alternative signaling receptor for GDNF family ligands. Cell 113, 867-79

269 Oo, T.F. et al. (2005) Anatomical basis of glial cell line-derived neurotrophic factor expression in the striatum and related basal ganglia during postnatal development of the rat. J. Comp. Neurol. 484, 57-67

270 Peng, C. et al. (2011) Pitx3 is a critical mediator of GDNF-induced BDNF expression in nigrostriatal dopaminergic neurons. J. Neurosci. 31, 12802-15

Figure 1: Neuronal activity promotes the proliferation of normal and malignant glial cells. Active cortical projection neurons (mauve) promote the proliferation of normal oligodendrocyte precursor cells (OPCs, green) and earlier neural precursor cells (NPCs) in the oligodendrocyte lineage. This activity-regulated response of myelin-forming precursor cells contributes to ongoing neuroplasticity during development and into adulthood [55]. While the molecular mediators of activity-regulated OPC proliferation remain to be elucidated, BDNF and glutamate are hypothesized to each play a role. Similar to the effects on healthy OPCs, active neurons drive highgrade glioma cell (red) proliferation in a range of glioma subtypes, including adult glioblastoma, oligodendroglioma, pediatric glioblastoma and pediatric diffuse intrinsic pontine glioma [68]. Neuronal activity-regulated glioma cell proliferation is mediated by a secreted form of neuroligin3 (sNLGN3), BDNF and glutamate [68,73,74]. Glioma cells in turn act on neurons to increase neuronal excitability and thus increase neuronal activity, an effect that is at least in part mediated by glutamate secretion by the glioma cells [76]. 
Figure 2: Peripheral innervation promotes glandular organ development and cancer progression. A) Glandular organogenesis depends on autonomic nerve innervation of the stem cell niche, and developing glands instruct proper nerve ingrowth (axon guidance) as illustrated in the submandibular salivary gland $[168,169]$. B) In pathological processes that mirror development, autonomic nerve innervation promotes prostate cancer progression [185] and prostate tumors instruct exuberant axonal sprouting and increased nerve density in the tumor microenvironment (axonogenesis; [184]). Ach = acetylcholine; Epi = epinephrine.

\section{Text Box 1: Development of the Central Nervous System (CNS)}

Development of the human CNS is a protracted process that spans decades. The brain and spinal cord form from neural stem cells (NSCs) of the germinal zone subjacent to the ventricles. During prenatal development, these stem cells (also called radial glial cells) first give rise to neurons, and after a "neurogenic to gliogenic switch" in mid-gestation, they begin to give rise to glial cells [245]. Establishment of the 6-layered cerebral cortex requires orderly migration of precursor cells along radial glial vertical fibers [246] and differentiation of layer-specific neurons in an "inside out" progression, with deep layers of cerebral cortex forming prior to superficial layers [245,247,248]. Neurogenesis involves neural stem cell proliferation, migration, and differentiation, with maturation of electrical excitability, outgrowth of axons and dendrites, and neurotransmitter specification. Following prenatal neurogenesis, astrogliogenesis and oligodendrogliogenesis occur in sequential but overlapping waves that extend well into postnatal life. Functional differentiation of oligodendrocytes to form myelin, or concentric wraps of oligodendrocyte membrane that provide electrical insulation of and metabolic support to axons, are predominantly postnatal processes that span at least the first three decades of human life [249,250]. In contrast to the cerebrum, brainstem and spinal cord, a great deal of cerebellar neuronal development occurs in the early postnatal period, with massive neurogenesis ( $\sim 38$ billion cerebellar granule cells) generated in the first year of human life [251]. Accordingly, cerebellar primitive neuroectodermal tumors, medulloblastomas, are among the most common solid cancers of infancy and early childhood. Cerebellar granule cell neurogenesis depends upon the proliferation, differentiation and survival of cerebellar granule precursor cells (GCPs), which emerge prenatally from the rhombic lip of the midbrain/hindbrain junction to the cerebellar anlage. 
Well into the postnatal period, GCPs proliferate in the external granule layer (EGL) of the developing cerebellum, then exit the cell cycle and migrate to the mature inner granule layer (IGL) via specialized radial fibers of Bergmann glia [252]; differentiating granule cell neurons in the IGL then project to and synapse on Purkinje cell dendrites in the molecular layer (Figure 1, Text Box 1). Additional cell types of the cerebellum arise from NPCs of the fourth ventricular zone.

Throughout life, new neuron generation continues in restricted regions of the central nervous system. Neurogenic NPCs may be a cell of origin for PNETs, ependymomas, and/or certain forms of glioma. Postnatal NSCs persist in the subventricular zone (SVZ) and in the dentate gyrus of the hippocampus (Figure 1, Text Box 1). SVZ NSCs give rise to neuroblasts that migrate along the rostral migratory stream [253-255] and differentiate into new neurons of the olfactory bulb and, in the human infant, of the prefrontal cortex [254]. SVZ precursors also give rise to OPCs in the mature brain [256], particularly in the context of injury [257]. Hippocampal stem cells residing in the subgranular zone (SGZ) of the dentate gyrus generate newborn neurons throughout life [258]. In adult humans, dentate gyrus neurogenesis results in $\sim 1400$ new hippocampal neurons daily [259].

OPCs are the most actively proliferative cell population in the adult brain of both rodents and humans (Figure 1, Text Box 1) [260,261] and precursors in the oligodendroglial lineage are thought to be the cell or origin for multiple subtypes of gliomas [10-14]. Robust new oligodendrocyte generation continues throughout life in rodents and non-human primates [262,263], and in humans continues at least into childhood [264]. In humans, non-human primates and rodents, myelination and myelin remodeling continues throughout life [262,264,265].

Figure 1, Text Box 1: Germinal zones of the postnatal human brain. After birth, active cell generation continues in several regions of the brain. The cerebellum continues robust development in infancy and early childhood (inset on right), with cerebellar granule cell precursors proliferating in the external granule layer (EGL, green) before migrating through the Purkinje cell (PC) layer to and differentiating in the internal granule layer (IGL). The cerebellum is a major site of pediatric brain tumors such as medulloblastoma, particularly in infancy and early childhood. In the subventricular zone (SVZ) of the lateral ventricles and the subgranule zone (SGZ) of the dentate gyrus in the hippocampus, neural stem and progenitor cells (green) persist throughout life. SVZ neural precursor cells give rise to new neurons of the olfactory bulb and (in infancy) frontal cortex as well as to new glial cells throughout life, particularly 
oligodendrocyte precursor cells (OPCs, green process-bearing cells). Hippocampal neural precursor cells continue to give rise chiefly to new dentate gyrus neurons throughout life. Throughout the brain, OPCs continue to proliferate and generate new oligodendrocytes. OPCs are shown in in the white matter (WM) of the corpus callosum; OPCs exist throughout both grey matter and white mater of the mature human brain. Both SVZ neural precursor cells and OPCs are putative cells of origin for multiple forms of glioma.

\section{Text Box 2: Neurotrophins}

The classical neurotrophin family, of which nerve growth factor (NGF) is the prototype, also includes brain-derived neurotrophic factor (BDNF), neurotrophin-3 (NT3) and neurotropin-4 (NT4). These neurotrophins bind to p75NTR, a Fas tumor necrosis factor receptor family member, and to one of the Trk tyrosine kinase receptors- either TrkA (NGF), TrkB (BDNF and NT4) or TrkC (NT3). TrK receptor tyrosine kinases signal to PKC, RAS/MAPK and PI3K pathways and are trophic in nature (for recent review, see [266]. In contrast, p75-mediated signaling is complex and can be either pro-apoptotic or trophic, depending on molecular context (for recent review, see [267]. NGF was the first neurotrophin discovered as responsible for sympathetic and sensory nerve growth and survival [95]. Subsequently, BDNF, NT-3 and NT-4 were also identified and implicated in a wide range of growth, maturation and plasticity-inducing effects throughout the nervous system and beyond.

A second family of neurotropic factors belonging to the TGF $\beta$ superfamily, the GDNF family of ligands (GFLs), includes glial-derived neurotrophin factor (GDNF), neurturin (NRTN), artemin (ARTN) and persephin (PSPN). Homodimeric ligands bind to receptor complexes composed of RET receptor tyrosine kinase and a GFR $\alpha$ family co-receptor; alternative receptors also exist [268]. GDNF was the first ligand discovered to promote the survival of midbrain dopaminergic neurons [96]. GDNF is expressed by striatal neurons, suggesting that in the healthy brain it is also a target-derived survival factor for midbrain DA neurons [269]. GDNF induces BDNF expression in midbrain dopaminergic neurons, which appears to contribute to its pro-survival effects [270]. The GFLs also play key roles in peripheral nervous system development.

\section{Text Box 3: Peripheral Nervous System}

The peripheral nervous system (PNS), derived largely from neural crest, is comprised of motor and autonomic efferent fibers and sensory afferent fibers. Most studies to date focused on the role of 
the autonomic nervous system (ANS), divided into adrenergic sympathetic and cholinergic parasympathetic arms, in development and cancer. Innervation of visceral organs, particularly by autonomic nerves, is extensive. Gastrointestinal system innervation, including 500 million enteric neurons located in myenteric and submucosal plexi, is so extensive that it is classified as its own division of the nervous system called the enteric nervous system (ENS). A number of recent studies demonstrated a direct role of peripheral innervation in numerous cancers, including prostate, gastric, pancreatic and skin tumors. In a broader range of cancers, there is evidence for the influence of neurotransmitters and neurotrophins in cancer progression, although the origin of these ligands may be from neural structures, normal stromal cells or the cancer cells themselves. The nervous system also influences cancers indirectly through regulation of hormone secretion via the hypothalamic-pituitary-adrenal axis; for example via elevation of circulating epinephrine and cortisol. This expansive and important topic is outside the scope of this review. 
Table summarizing neural molecules in cancer_122816

\begin{tabular}{|c|c|c|c|c|}
\hline Neural Signal & Tumor Type & Demonstrated Function & Descriptive Observations & Refs \\
\hline \multirow[t]{2}{*}{$\begin{array}{l}\text { Glutamatergic } \\
\text { signaling }\end{array}$} & $\begin{array}{c}\text { Medulloblastoma } \\
\text { Osteosarcoma }\end{array}$ & $\begin{array}{l}\text { mGlur4 agonism reduces proliferation } \\
\text { in vitro; decreases progression in vivo }\end{array}$ & $\begin{array}{l}\text { mGlur4 correlates inversely with } \\
\text { histological anaplasia, dissemination and } \\
\text { clinical outcome } \\
\text { mGluR4 was found to be expressed in } \\
\text { osteosarcoma }(\sim 20 \%) \text { and benign giant } \\
\text { cell tumors of bone }(\sim 40 \%) \text {, and } \\
\text { expression correlated positively with } \\
\text { overall survival in osteosarcoma }\end{array}$ & $154-156$ \\
\hline & Glioma & $\begin{array}{l}\text { Secretion of glutamate by tumor cells } \\
\text { increases cell proliferation, survival } \\
\text { and migration/invasion can be } \\
\text { mediated by calcium-permeable } \\
\text { AMPA receptor stimulation }\end{array}$ & & $70-76$ \\
\hline $\begin{array}{l}\text { GABAergic } \\
\text { signaling }\end{array}$ & $\begin{array}{c}\text { Medulloblastoma } \\
\text { Glioma }\end{array}$ & $\begin{array}{l}\text { Activation of } \mathrm{GABA}_{\mathrm{A}} \text { receptors can be } \\
\text { either depolarizing or hyperpolarizing }\end{array}$ & $\begin{array}{l}\text { Group } 3 \text { medulloblastomas exhibit } \\
\text { increased expression of GABA receptor } \\
\alpha 5 \\
\text { Tends to be present in lower grade } \\
\text { gliomas (grade II and III astrocytomas) } \\
\text { and in oligodendrogliomas rather than } \\
\text { GBM (grade IV) }\end{array}$ & 77,78 \\
\hline $\begin{array}{l}\text { Adrenergic } \\
\text { signaling } \\
\text { receptors }\end{array}$ & $\begin{array}{c}\text { Ewing sarcoma } \\
\text { and osteosarcoma } \\
\text { Prostate cancer }\end{array}$ & $\begin{array}{l}\text { Chemical sympathectomy or genetic } \\
\text { deletion of stromal } \beta 2-\text { and } \beta 3- \\
\text { adrenergic receptors results in poor } \\
\text { tumor engraftment and growth }\end{array}$ & $\beta$-adrenergic receptors are expressed & 185 \\
\hline
\end{tabular}




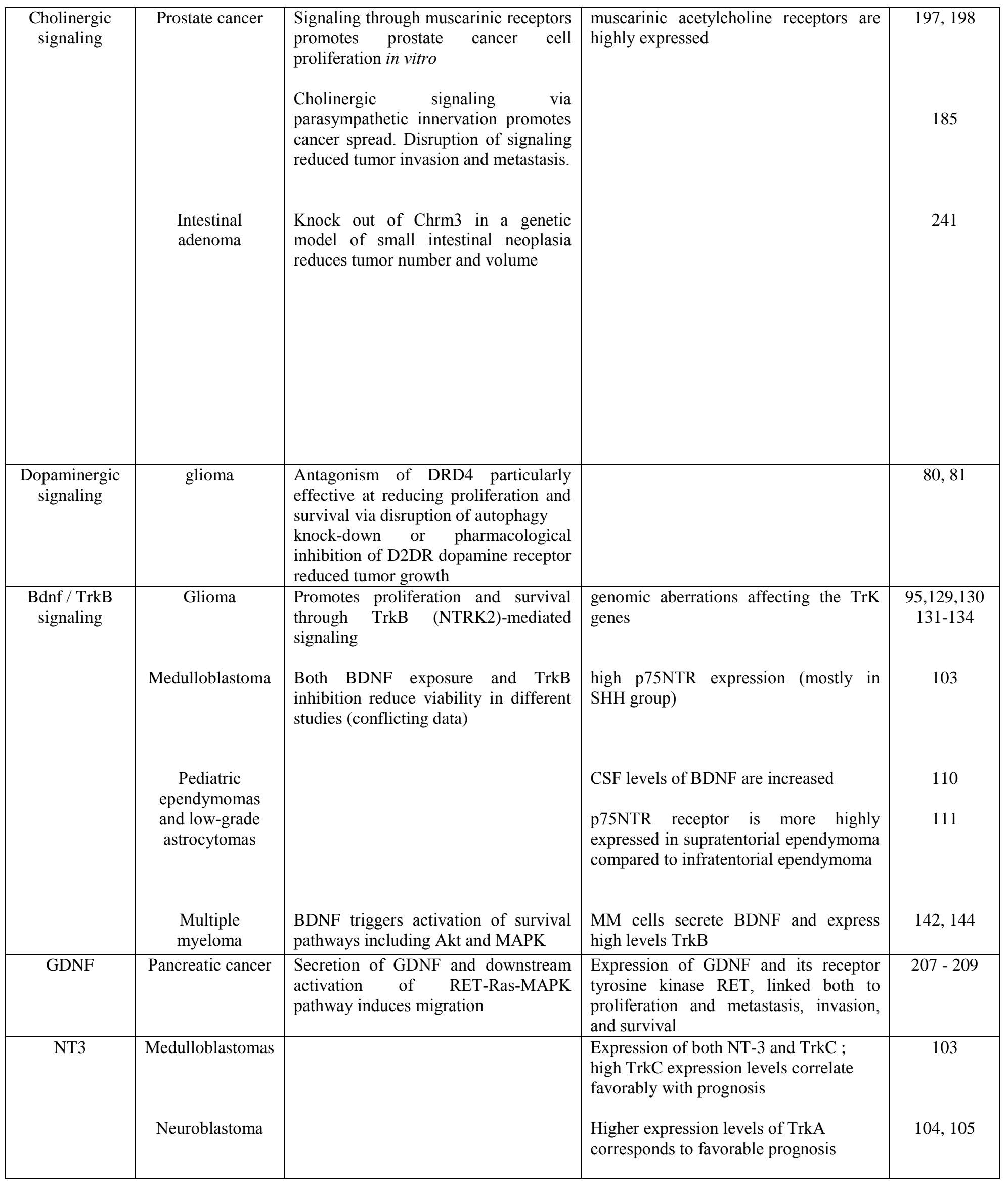




\begin{tabular}{|c|c|c|c|c|}
\hline $\begin{array}{c}\text { NGF/ } \\
\text { Pro-NGF }\end{array}$ & $\begin{array}{c}\text { Melanoma } \\
\text { Prostate cancer } \\
\text { Pancreatic cancer }\end{array}$ & $\begin{array}{l}\text { Autocrine/paracrine neurotrophin } \\
\text { signaling promotes the proliferation } \\
\text { and migration of melanoma cells }\end{array}$ & $\begin{array}{l}\text { Expression of a range NGF family } \\
\text { neurotrophin receptors and ligands } \\
\text { NGF correlated positively with the } \\
\text { Gleason score } \\
\text { NGF increased in pancreatic } \\
\text { adenocarcinoma } \\
\text { Both receptors of NGF, TrkA and } \\
\text { p75NTR, are associated with perineural } \\
\text { invasion. p75NTR is associated with a } \\
\text { favorable prognosis, while TrkA is } \\
\text { associated with poor prognosis. }\end{array}$ & $\begin{array}{c}187 \\
206 \\
210,211\end{array}$ \\
\hline Nlgn3 & $\begin{array}{l}\text { High-grade } \\
\text { glioma }\end{array}$ & Secreted NLGN3 promotes growth & $\begin{array}{l}\text { Genomic aberrations across multiple } \\
\text { cancer type }\end{array}$ & 68 \\
\hline $\begin{array}{c}\text { Novel } \\
\text { neurotrophin- } \\
\text { 1/NNT1/BSF3 }\end{array}$ & $\begin{array}{l}\text { Multiple } \\
\text { myeloma }\end{array}$ & $\begin{array}{l}\text { Linked to growth and survival of } \mathrm{MM} \\
\text { in vitro }\end{array}$ & & $140-142$ \\
\hline
\end{tabular}




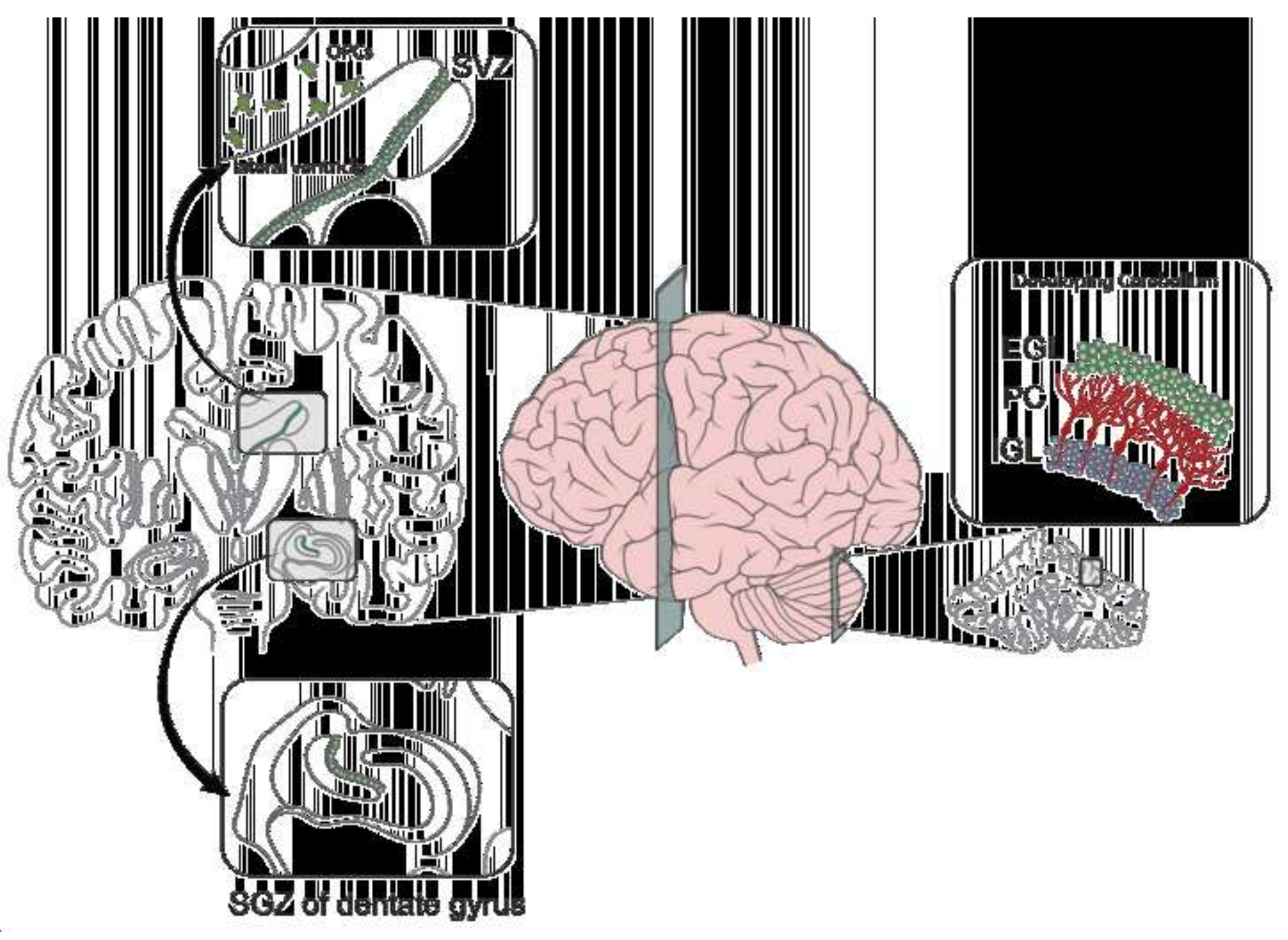




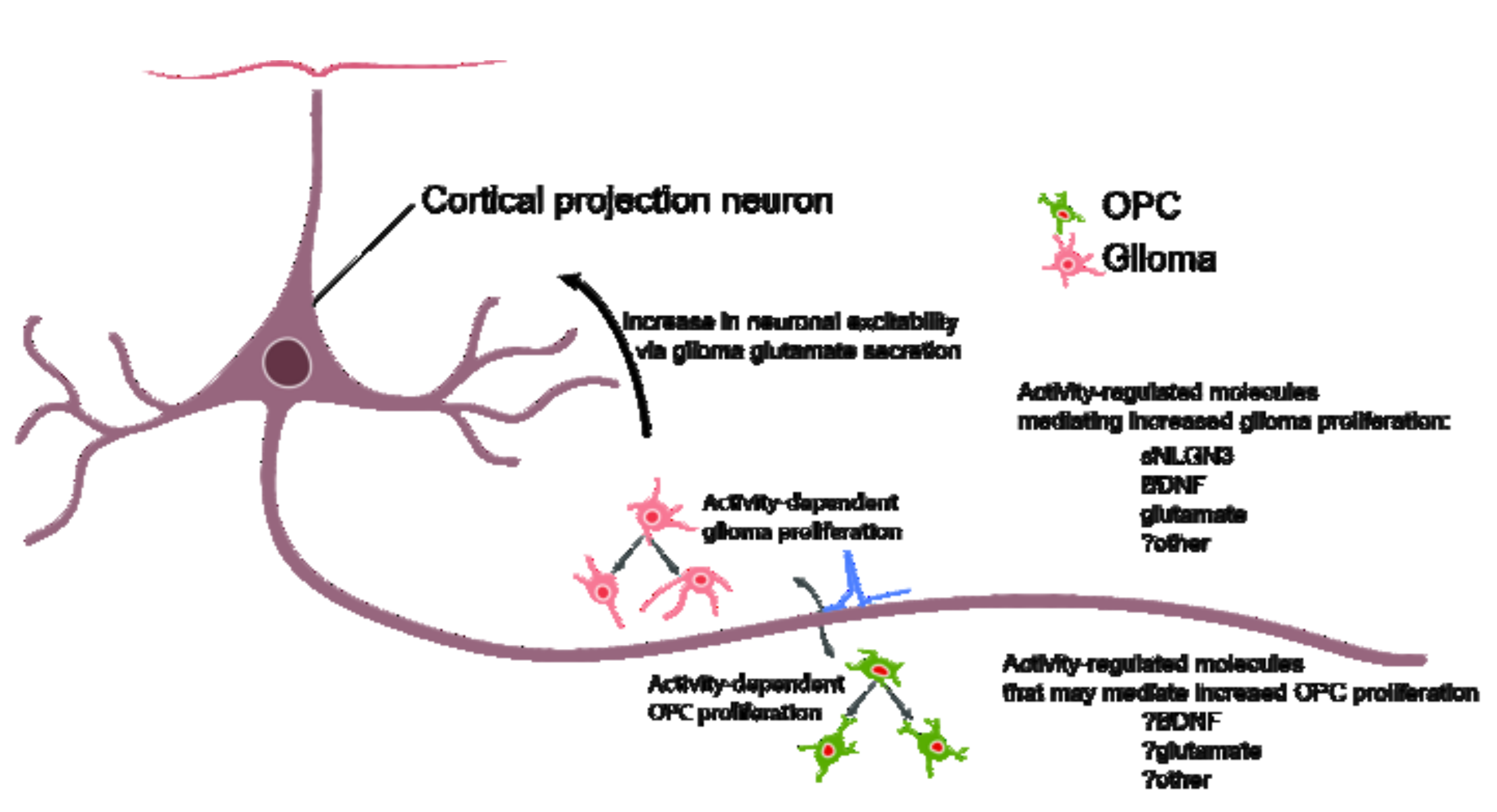



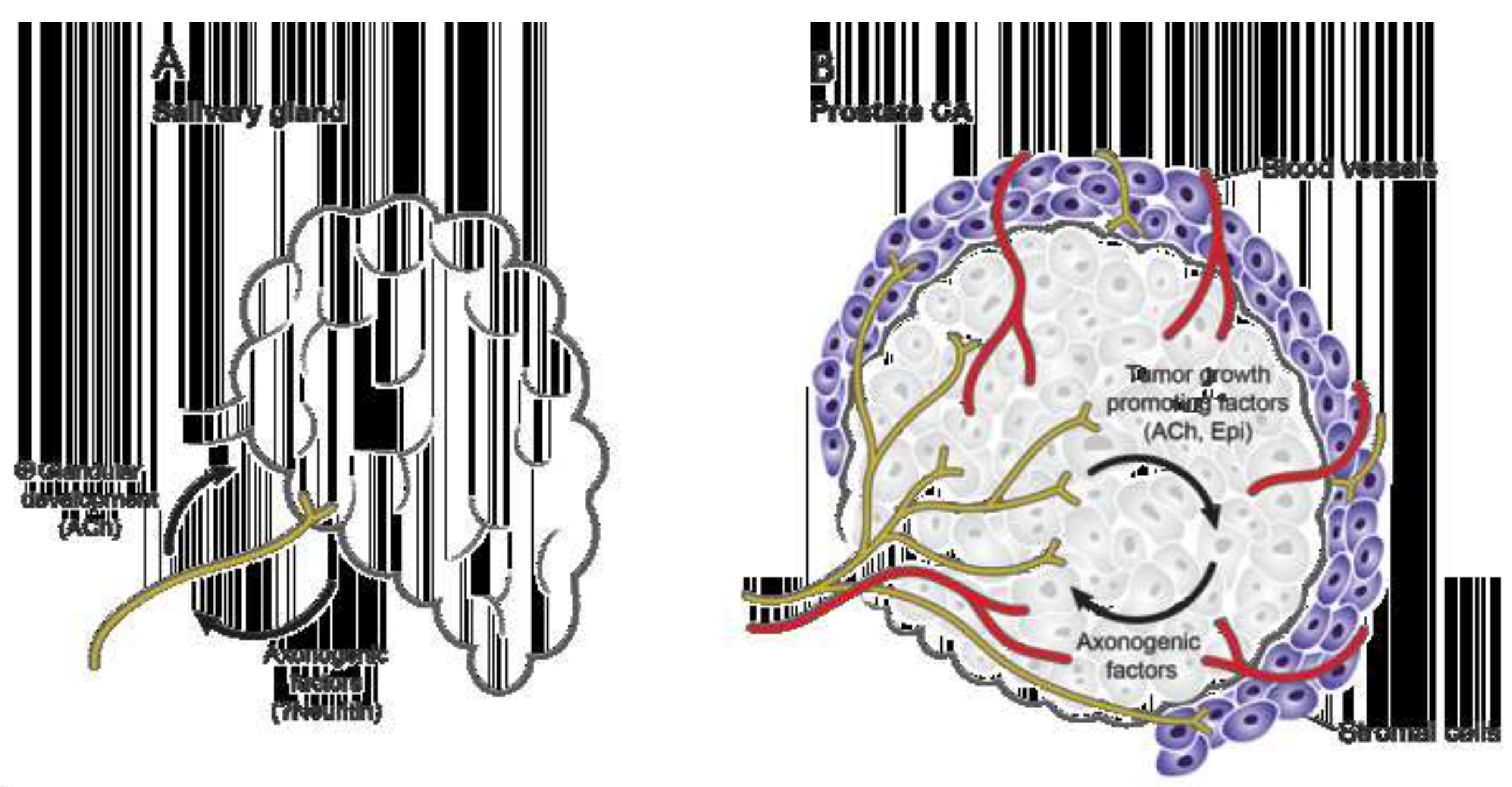